\title{
THE Lip-lip EQUALITY IS STABLE UNDER BLOW-UP
}

\author{
ANDREA SCHIOPPA
}

\begin{abstract}
We show that at generic points blow-ups/tangents of differentiability spaces are still differentiability spaces; this implies that an analytic condition introduced by Keith as an inequality (and later proved to actually be an equality) passes to tangents. As an application, we characterize the $p$-weak gradient on iterated blow-ups of differentiability spaces.
\end{abstract}

\section{Contents}

1. Introduction

2. Background Material 5

3. Blow-up of Weaver Derivations $\quad 12$

4. A local approach to fail differentiability 19

5. Blow-ups of differentiability spaces 21

References

\section{INTRODUCTION}

Background. The study of the geometric and analytic properties of metric spaces is a topic which has grown into many different trends, and is probably as old as the study of fractal subsets of $\mathbb{R}^{n}$ and of Carnot groups. In the last 15 years, a trend which has attracted growing interest is the study of metric measure spaces which admit an abstract Poincaré inequality in the sense of [HK98], and which we will call PI-spaces.

Intuitively, PI-spaces allow to formulate notions of first-order calculus, an intuition that was made more precise when [Che99] proved that PI-spaces satisfy a generalized version of the classical Rademacher Theorem about the a.e. differentiability of real-valued Lipschitz functions. In particular, Cheeger's result allows to associate to a PI-space $(X, \mu) \mu$-measurable tangent / cotangent bundles $T X / T^{*} X$, the fibres of $T^{*} X$ being generated by "differentials" of Lipschitz functions. It is worth to point out that Cheeger's generalization of Rademacher's Theorem does not put $T X$ and $T^{*} X$ on an equal footing, e.g. derivatives are not explicitly constructed and are not related to differentiation along Lipschitz curves. Essentially, differentiability is formulated in terms of finite-dimensionality results for certain spaces of (asymptotically) harmonic functions. Note that even though this approach might

2010 Mathematics Subject Classification. 53C23, 46J15, 58C20, 31E05.

Key words and phrases. derivation, differentiation, Lipschitz functions, Sobolev spaces.

The author was supported by the "ETH Zurich Postdoctoral Fellowship Program and the Marie Curie Actions for People COFUND Program" and the European Research Council grant n. 291497. 
look at first counterintuitive, it fits with the idea that coordinate functions generate the cotangent bundle of a Riemannian manifold as other functions admit a first-order Taylor expansion with respect to the coordinates, and moreover in Riemannian geometry there are important results on finite-dimensionality of spaces of harmonic functions whose proofs share some similarities with Cheeger's argument (see for instance [CM97]).

Today metric measure spaces which satisfy the conclusion of Cheeger's Differentiation Theorem are either said to admit a (strong) measurable differentiable structure [Kei02, Kei04], or to be (Lipschitz) differentiability spaces [Bat12, Bat15]; in the following we will use the latter terminology.

In his $\mathrm{PhD}$ thesis, Keith [Kei02, Kei04] introduced a new analytic condition, the Lip-lip inequality, and proved that doubling metric measure spaces $(X, \mu)$ satisfying it are differentiability spaces. It seems that the idea of "generalizing" Cheeger's Differentiation Theorem using a Lip-lip inequality stems from the fact that in PIspaces Cheeger had proven a Lip-lip equality. We use "generalize" to refer to Keith's work because as of today there seem to be no examples of differentiability spaces which cannot be partitioned into countable unions of subsets, each of which admits a measure-preserving biLipschitz embedding into some PI-space.

Besides providing a theoretical framework for first-order calculus, the idea of differentiating Lipschitz functions has proven useful in the study of metric embeddings $F: X \rightarrow B$ where $B$ is a Banach space, in particular when either $B$ has the RadonNikodym property (i.e. an RNP Banach space) (see for instance[CK09]), or when $B=L^{1}$ (see for instance [CK10a, CK10b, CKN11, CK13]). In connection with embeddings into RNP-Banach spaces, Cheeger and Kleiner [CK09] showed that if $(X, \mu)$ is a PI-space the fibres of $T X$ are spanned by "tangent vectors" to Lipschitz curves. Putting $T X$ and $T^{*} X$ on a complete equal footing has required substantial effort: Bate's beautiful work [Bat12, Bat15] on Alberti representations in differentiability spaces, which was partly motivated by a deep structure theory for measures and sets in $\mathbb{R}^{n}$ developed by Alberti, Csörnyei and Preiss [ACP05, ACP10], and the formulation of metric differentiation for differentiability spaces [CKS15], which was partly motivated by unpublished results of Cheeger and Kleiner on metric differentiation in PI-spaces, and unpublished results of mine on prescribing the norms on $T X$ and $T^{*} X$.

Strikingly, Bate [Bat12, Bat15] was able to show that each differentiability space $(X, \mu)$ can be partitioned into countably many pieces, each of which is a doubling metric measure space admitting a Lip-lip inequality. Later we showed [Sch14b, Sch13] that the Lip-lip inequality always self-improves to an equality (see also [CKS15] for another argument); this might be interpreted as saying that the Lip-lip equality provides an asymptotically quantitative characterization of differentiability spaces; however, there is a more precise result in terms of the quantitative characterization of the local norm for Weaver forms (Theorem 2.29) which will be used in this paper.

In connection with these results, a topic of major interest is trying to understand the infinitesimal structure of differentiability, and even PI-spaces. It should be pointed out that, as of today, the set of known models for the infinitesimal geometry of PI-spaces is rather limited, and is thus hard to come up with "plausible" conjectures. For example, while the results in [CKS15] show structural similarities between PI-spaces and differentiability spaces (e.g. one might conjecture 
that tangents/blow-ups of differentiability spaces are PI spaces), the examples constructed in [Sch15] suggest that there might be differentiability spaces $(X, \mu)$ whose blow-ups are never PI-spaces; moreover, this phenomenon might even depend on the measure class of $\mu$. While finishing this paper, we have learned from Bate and Li [BL15] that they have studied the class of differentiability spaces for which differentiation of RNP-valued functions (in addition to that of real-valued ones) holds, and have characterized them in terms of an "infinitesimal accessibility" condition. One possibility is that differentiability implies RNP-differentiability, and then extending the results of [CK09] to general differentiability spaces might give a route to answer some questions raised in [CKS15]. Another possibility is that differentiability spaces are organized in a sort of "hierarchy" depending on which Banach-valued Lipschitz maps are differentiable. For instance, the argument in [BL15] uses crucially differentiability in the $l_{1}$-sum of a sequence of finite-dimensional spaces $\left\{l_{\infty}^{n_{k}}\right\}_{k}$ where $n_{k} \nearrow \infty$, and it does not seem clear how one would recover the result in [BL15] assuming, say, differentiability of $l_{2}$-valued maps.

Results. The main result of this paper states that if $(X, \mu)$ is a differentiability space, then at $\mu$-a.e. $x \in X$ any blow-up $\left(Y_{x}, \nu_{x}\right)$ of $(X, \mu)$ is still a differentiability space. The precise statement is Theorem 5.1.

The question of whether blow-ups of differentiability spaces are still differentiability spaces has been around since Keith introduced the Lip-lip inequality. This question should be compared with the easier case where $(X, \mu)$ is a PI-space: then any blow-up is still a PI-space. This follows from the stability of the Poincaré inequality (with uniform constants) under measured Gromov-Hausdorff convergence, which can be seen using Keith's elegant characterization of PI-spaces in terms of moduli of families of curves [Kei03]. Essentially, the argument reduces to the uppersemicontinuity of modulus (which is dual to the lower-semicontinuity of length). On the other hand, in the category of differentiability spaces one cannot expect all blow-ups to be differentiability spaces. For example, any subset $S \subset \mathbb{R}^{n}$ of positive Lebesgue measure is a differentiability space (restricting the Lebesgue measure), but blow-ups are differentiability spaces (explicitly, copies of $\mathbb{R}^{n}$ with Lebesgue measure) only at generic points. Moreover, at the moment there is no "good geometric" characterization of differentiability spaces that one might pass to the limit; one has to work directly with the definition of a differentiability space by showing that if at $\mu$-a.e. point $x$ there is some tangent $\left(Y_{x}, \nu_{x}\right)$ which is not a differentiability space, then $(X, \mu)$ is not a differentiability space. This can be done from two equivalent points of view: [Bat12, Sec. 4] choosing a candidate chart and producing a non-differentiable function for that chart, [Sch13, Sec. 5.3] showing that the space of "germs" of Lipschitz functions is infinite-dimensional.

The methodology that we propose to tackle this problem has two components: "lifting" and "gluing". Lifting means to find a suitable "bad" function $g_{x}$ on a blow-up $\left(Y_{x}, \nu_{x}\right)$, and then lift it to a bad function $f_{x}$ on $(X, \mu)$. Unfortunately, $f_{x}$ will be bad only at some locations near $x$ and at some scales (which can be small but are bounded away from 0 ); thus it is then necessary to glue several $f_{x}$ 's together. It is worth to point out that lifting depends on the structure theory for Weaver derivations developed in [Sch14b, Sch13]. In fact, $\left(Y_{x}, \nu_{x}\right)$ is not a differentiability space, but there is still a decent theory (in particular finite-dimensionality) for the $L^{\infty}$-modules of Weaver derivations and forms. Another key ingredient in lifting 
is Preiss' phenomenon (Theorem 3.18) that shifted rescalings of blow-ups are still blow-ups.

Gluing consists in combining several $f_{x}$ 's together. The proposed approach is based on the idea of "tile" (see Section 4): for each $x$ one has to produce several $f_{x}$ 's at several scales converging to 0 , and then one applies the Vitali Covering Lemma to join the pieces together. Intuitively, we are using measure theory (e.g. the measuretheoretic statement that $(X, \mu)$ is finite-dimensional) to select the pieces that can be glued together; in some sense, the construction can't be "deterministic" because Lipschitz functions are rather rigid and $(X, \mu)$ is a generic doubling metric measure space.

We mention some further directions of research. We are able to show that for iterated blow-ups of differentiability spaces the analytic dimension does not increase (see Lemma 5.59). It seems plausible to conjecture that one does not need to take iterated blow-ups: at the moment we are able to prove this only under additional assumptions on $(X, \mu)$ (e.g. when $(X, \mu)$ biLipschitz embedds in Carnot groups or "nice" Banach spaces), and thus do not include these partial results here. Essentially, there seems to be a technical obstacle in directly applying the "lifting" method in the form proposed here. This question is also related to wheteher the results in [CK09] extend to general differentiability spaces, and on Lusin-like properties for Weaver derivations that will be discussed elsewhere.

As an application, we obtain a characterization of the $p$-weak gradient of a Lipschitz function $f$ on regular differentiability spaces (Definition 5.57) which arise as iterated blow-ups of differentiability spaces.

This is related to another trend in analysis on metric spaces, where people have tried to formulate generalizations of Sobolev/BV spaces and gradients. One possible approach starts with the idea [HK98] of an upper gradient for a function $f$, which is essentially an upper bound on the norm of "what the gradient of $f$ should be". Then there have been several proposals for "what the norm of the gradient should be": a variational one due to [Che99], one motivated by quasiconformal geometry and moduli of curves due to Koskela, MacManus and Shanmugalingam [KM98, Sha00], and two motivated by optimal transport due to Ambrosio, Gigli and Savaré [AGS13]. Optimal transport allows to show [AGS13] that all these approaches are equal under mild assumptions on $(X, \mu)$, e.g. assuming $X$ to be complete and separable, and $\mu$ to be finite on bounded sets. In this paper we work with the $p$-weak gradient of [AGS13] because it is easier to relate to the existence of Alberti representations using the notion of test plan.

Asking for a characterization of the $p$-weak gradient on differentiability spaces is not a good question because positive measure subsets of differentiability spaces are still differentiability spaces, making the notion of $p$-weak gradient often vacuous (e.g. considering a Vitali-Cantor set). However, an interesting question is to ask for a characterization of the $p$-weak gradient on blow-ups of differentiability spaces. We show (Theorem 5.63) that for regular differentiability spaces the $p$-weak gradient of a Lipschitz function $f$ coincides with the asymptotic Lipschitz constant, and is hence independent of the exponent $p$ : this generalizes a previous result [Che99] for PI-spaces. Note however, that for PI-spaces the $p$-weak gradient coincides with the asymptotic Lipschitz constant only if $p$ belongs to the range of exponents for which the Poincaré inequality holds: this has been shown by recent examples [DS15]. This 
is not the case for regular differentiability spaces: the $p$-weak gradient does not depend on $p$. One can explain the examples [DS15] in terms of putting "bad weights" on the lines corresponding to Alberti representations, but these bad weights become again "nice" by passing to tangent measures. We point out that Theorem 5.63 has an analogue in the BV category, see Remark 5.79 (see [ADM14] for extensions of [AGS13] to the BV category).

As a historical note, we point out that the notion of regular differentiability space refines and generalizes the notion of generalized Minkowski space in [Che99, Sec. 11].

Organization. In Section 2 we discuss background material on Weaver derivations, Alberti representations, my $\mathrm{PhD}$ thesis, differentiability spaces and the $p$ weak gradient. The presentation is a bit brisk, so we invite the interested reader to consult the references therein.

In Section 3 we first discuss (subsection 3.1) variants of Gromov-Hausdorff convergence, the purpose being mainly to establish some terminology. The substantial result from this subsection that we will use is the aforementioned Preiss' phenomenon, Theorem 3.18 [Pre87, LD11, GMR15]. We then move on (subsection 3.2) to explain how rescalings affect the modules of derivations and forms. We conclude this section with a generalization of a result [CKS15, Sec. 7] to the category of Weaver derivations. We point out that this result to blow-up Weaver derivations has other applications, e.g. to the infinitesimal structure of metric currents in $\mathbb{R}^{n}$, that will be discussed elsewhere.

Section 4 contains our proposal to implement the "gluing" part of the argument; this is more general than what we really use here, because we end up working with "cubical" tiles [Chr90, HK12]. Using other geometries for tiles might lead to a better understanding of the geometry of blow-ups; for example, Preiss and I discussed sometime ago what are essentially "long cylindrical" tiles to exclude factorizations of the form $Y \times \mathbb{R}^{n}$ in blow-ups of differentiability spaces.

In subsection 5.1 it is shown that generic blow-ups of differentiability spaces are still differentiability spaces by proving the contrapositive statement; the first 6 steps of Theorem 5.1 correspond to "lifting". Subsection 5.2 discusses the characterization of the $p$-weak gradient on regular differentiability spaces; the key step is Lemma 5.49 which associates test plans to "nice" Alberti representations.

Notational conventions. We use the convention $a \approx b$ to say that $a / b, b / a \in$ $\left[C^{-1}, C\right]$ where $C$ is a universal constant; when we want to highlight $C$ we write $a \approx_{C} b$. We similarly use notations like $a \lesssim b$ and $a \gtrsim_{C} b$.

Acknowledgements. This work would have not been possible without the many conversations that I had with David Preiss, who generously invited me to visit the University of Warwick; I also wish to thank the people in the analysis group, in particular Daniel Seco, for the hospitality I received.

I also thank the anonymous referee for reading the manuscript very carefully and for pointing out an issue with the way measures were normalized in the first version of the preprint.

\section{Background Material}


2.1. Weaver derivations. For more information we refer the reader to [Wea00]. An $L^{\infty}(\mu)$-module $M$ is a Banach space $M$ which is also an $L^{\infty}(\mu)$-module and such that for all $(m, \lambda) \in M \times L^{\infty}(\mu)$ one has:

$$
\|\lambda m\|_{M} \leq\|\lambda\|_{L^{\infty}(\mu)}\|m\|_{M}
$$

Among $L^{\infty}(\mu)$-modules a special rôle is played by $L^{\infty}(\mu)$-normed modules:

Definition 2.2 (Normed modules). An $L^{\infty}(\mu)$-module $M$ is said to be an $L^{\infty}(\mu)$ normed module if there is a map

$$
|\cdot|_{M, \text { loc }}: M \rightarrow L^{\infty}(\mu)
$$

such that:

(1) For each $m \in M$ one has $|m|_{M \text {,loc }} \geq 0$;

(2) For all $c_{1}, c_{2} \in \mathbb{R}$ and $m_{1}, m_{2} \in M$ one has:

$$
\left|c_{1} m_{1}+c_{2} m_{2}\right|_{M, \text { loc }} \leq\left|c_{1}\right|\left|m_{1}\right|_{M, \text { loc }}+\left|c_{2}\right|\left|m_{2}\right|_{M, \text { loc }}
$$

(3) For each $\lambda \in L^{\infty}(\mu)$ and each $m \in M$, one has:

$$
|\lambda m|_{M, \text { loc }}=|\lambda||m|_{M, \text { loc }}
$$

(4) The local seminorm $|\cdot|_{M \text {, loc }}$ can be used to reconstruct the norm of any $m \in M$ :

$$
\|m\|_{M}=\left\||m|_{M, \text { loc }}\right\|_{L^{\infty}(\mu)} .
$$

Definition 2.7 (Weaver derivation). A derivation $D: \operatorname{Lip}_{\mathrm{b}}(X) \rightarrow L^{\infty}(\mu)$ is a weak* continuous, bounded linear map satisfying the product rule:

$$
D(f g)=f D g+g D f .
$$

Note that the product rule implies that $D f=0$ if $f$ is constant. The collection of all derivations $X(\mu)$ is an $L^{\infty}(\mu)$-normed module [Wea00, Thm. 2] and the corresponding local norm will be denoted by $|\cdot|_{X(\mu) \text {,loc }}$. Note also that $X(\mu)$ depends only on the measure class of $\mu$.

Remark 2.9. Consider a Borel set $U \subset X$ and a derivation $D \in X(\mu\llcorner U)$. The derivation $D$ can be also regarded as an element of $\mathcal{X}(\mu)$ by extending $D f$ to be 0 on $X \backslash U$. In particular, the module $X(\mu\llcorner U)$ can be naturally identified with the submodule $\chi_{U} X(\mu)$ of $\mathcal{X}(\mu)$.

Derivations are local in the following sense [Wea00, Lem. 27]):

Lemma 2.10 (Locality of Derivations). If $U$ is $\mu$-measurable and if $f, g \in \operatorname{Lip}_{\mathrm{b}}(X)$ agree on $U$, then for each $D \in \mathcal{X}(\mu), \chi_{U} D f=\chi_{U} D g$.

Note that locality allows to extend the action of derivations on Lipschitz functions so that if $f \in \operatorname{Lip}(X)$ and $D \in X(\mu), D f$ is well-defined (see Remark 2.115 in [Sch13]). We now pass to consider some algebraic properties of $X(\mu)$.

In general, even if the module $\mathcal{X}(\mu)$ is finitely generated, it is not free. Nevertheless, it is possible to obtain a decomposition into free modules over smaller rings [Wea00, Sch14c] :

Theorem 2.11 (Free Decomposition). Suppose that the module $X(\mu)$ is finitely generated with $N$ generators. Then there is a Borel partition $X=\bigcup_{i=0}^{N} X_{i}$ such that, if $\mu\left(X_{i}\right)>0$, then $X\left(\mu\left\llcorner X_{i}\right)\right.$ is free of rank $i$ as an $L^{\infty}\left(\mu\left\llcorner X_{i}\right)\right.$-module. A basis of $X\left(\mu\left\llcorner X_{i}\right)\right.$ will be called a local basis of derivations. 
Remark 2.12. In particular, Theorem 2.11 can be applied if one knows an upper bound on the index of $\mathcal{X}(\mu)$ which is defined as follow:

$$
\begin{array}{r}
\text { index of } \mathcal{X}(\mu)=\sup \{n \in \mathbb{N}: \exists U \text { Borel: } X(\mu\llcorner U) \text { contains } n \text {-independent } \\
\text { elements (over } \left.L^{\infty}(\mu\llcorner U))\right\} .
\end{array}
$$

In many applications in analysis on metric spaces the assumption that $X(\mu)$ has finite index (and is hence finitely generated) is not restrictive: for example it holds if either $\mu$ or $X$ are doubling (see Corollary 5.136 in [Sch13]).

We now recall the notion of 1 -forms which are dual to derivations.

Definition 2.14. The module of 1 -forms $\mathcal{E}(\mu)$ is the dual module of $X(\mu)$, i.e. it consists of the bounded module homomorphisms $X(\mu) \rightarrow L^{\infty}(\mu)$. The module $\mathcal{E}(\mu)$ is an $L^{\infty}(\mu)$-normed module and the local norm will be denoted by $|\cdot|_{\varepsilon(\mu) \text {,loc }}$.

To each $f \in \operatorname{Lip}_{\mathrm{b}}(X)$ one can associate the 1 -form $d f \in \mathcal{E}(\mu)$ by letting:

$$
\langle d f, D\rangle=D f \quad(\forall D \in X(\mu)) ;
$$

the map $d: \operatorname{Lip}_{\mathrm{b}}(X) \rightarrow \mathcal{E}(\mu)$ is a weak* continuous 1-Lipschitz linear map satisfying the product rule $d(f g)=g d f+f d g$.

Note that because of Lemma 2.10 one can extend the domain of $d$ to $\operatorname{Lip}(X)$ so that if $f$ is Lipschitz, $d f$ is a well-defined element of $\mathcal{E}(\mu)$ and $\|d f\|_{\mathcal{E}(\mu)} \leq \mathbf{L}(f)$, where $\mathbf{L}(f)$ denotes the global Lipschitz constant of $f$.

2.2. Alberti representations. Alberti representations (without this name) were introduced in [Alb93]; we invite the reader to consult [Bat15, Sch13, CKS15] for more information.

Definition 2.16 (Fragments and Curves). A fragment in $X$ is a Lipschitz map $\gamma: C \rightarrow X$, where $C \subset \mathbb{R}$ is closed. The set of fragments in $X$ will be denoted by Frag $(X)$. We now discuss the topology on $\operatorname{Frag}(X)$; let $F(\mathbb{R} \times X)$ denote the set of closed subsets of $\mathbb{R} \times X$ with the Fell topology [Kec95, (12.7)] ; we recall that a basis of the Fell topology consists those sets of the form:

$$
\left\{F \in F(\mathbb{R} \times X): F \cap K=\emptyset, F \cap U_{i} \neq \emptyset \text { for } i=1, \ldots, n\right\},
$$

where $K$ is a compact subset of $\mathbb{R} \times X$, and $\left\{U_{i}\right\}_{i=1}^{n}$ is a finite collection of open subsets of $\mathbb{R} \times X$. Each fragment $\gamma$ can be identified with an element of $F(\mathbb{R} \times X)$ and thus Frag $(X)$ will be topologized as a subset of $F(\mathbb{R} \times X)$. We will use fragments to parametrize 1-rectifiable subsets of $X$.

An important subset of $\operatorname{Frag}(X)$ consists of the Lipschitz curves Curves $(X)$, which is the set of those $\gamma \in \operatorname{Frag}(X)$ whose domain is a (possibly unbounded) closed subinterval of $\mathbb{R}$. Given an interval $I \subset[0,1]$ we denote by $\operatorname{Curves}(X, I) \subset$ $\operatorname{Curves}(X)$ the set of those $\gamma \in \operatorname{Curves}(X)$ whose domain is contained in $I$.

Definition 2.18 (Alberti representations). Let $\mu$ be a Radon measure on $X$. An Alberti representation of $\mu$ is a pair $\mathcal{A}=[Q, w]$ where $Q$ is a Radon measure on $\operatorname{Frag}(X)$ and $w$ a Borel function $w: X \rightarrow[0, \infty)$ such that:

$$
\mu=\int_{\operatorname{Frag}(X)} w \cdot \gamma_{\#}\left(\mathcal{L}^{1}\llcorner\operatorname{dom} \gamma) d Q(\gamma)\right.
$$

where the integral is interpreted in the weak* sense. We say that $\mathcal{A}$ is $C$-Lipschitz (resp. [C,D]-biLipschitz) if $Q$ is concentrated on the set of $C$-Lipschitz (resp. [C, $D]$ biLipschitz) fragments. 
Definition 2.20 (Speed of fragments). Let $\gamma \in \operatorname{Frag}(X)$; then for $\mathcal{L}^{1}$-a.e. $t \in \operatorname{dom} \gamma$ the limit:

$$
\lim _{\operatorname{dom} \gamma t^{\prime} \rightarrow t} \frac{d\left(\gamma\left(t^{\prime}\right), \gamma(t)\right)}{\left|t^{\prime}-t\right|}
$$

exists, is denoted by $\operatorname{md} \gamma(t)$ and is called the metric differential of $\gamma$ at $t$.

Definition 2.22 (Speed of Alberti representations). Let $\mathcal{A}=[Q, w]$ be an Alberti representation, and let $\sigma: X \rightarrow[0, \infty)$ be a Borel function and $f: X \rightarrow \mathbb{R}$ be a Lipschitz function. We say that $\mathcal{A}$ has $f$-speed $\geq \sigma$ (resp. $\leq \sigma)$ if for $Q$-a.e. $\gamma$ one has that for $\mathcal{L}^{1}$-a.e. $t \in \operatorname{dom} \gamma$ :

$$
(f \circ \gamma)^{\prime}(t) \geq \sigma(\gamma(t)) \mathrm{md} \gamma(t) \quad(\text { resp. } \leq \sigma(\gamma(t)) \operatorname{md} \gamma(t)) .
$$

2.3. The correspondence between derivations and Alberti representations. We invite the reader to consult [Sch14b, Sch13] for more information. The following definition follows from Theorem 3.11 in [Sch13].

Definition 2.24 (Derivation associated to an Alberti representation). Let $\mathcal{A}=$ $[Q, w]$ be a $C$-Lipschitz Alberti representation of a measure $\nu \ll \mu$; then the formula:

$$
\begin{aligned}
& \int_{X} g D_{\mathcal{A}} f d \nu=\int_{\operatorname{Frag}(X)} d Q(\gamma) \int_{\operatorname{dom} \gamma}(w g) \circ \gamma(t)(f \circ \gamma)^{\prime}(t) d t \\
&\left(\forall(g, f) \in C_{c}(X) \times \operatorname{Lip}_{\mathrm{b}}(X)\right)
\end{aligned}
$$

defines a derivation $D_{\mathcal{A}} \in \mathcal{X}(\nu) \subset \mathcal{X}(\mu)$ with $\left\|D_{\mathcal{A}}\right\|_{X(\mu)} \leq C$.

The following theorem summarizes some results in [Sch14b, Sch13] that we will use.

Theorem 2.26 (Correspondence between derivations and representations). Let $\mu$ be a Radon measure on a complete separable metric measure space $(X, \mu)$. Then:

(IndBound): If $\mu$ or $X$ are doubling (with constant $C$ ), then the index of $X(\mu)$ is $\leq\left\lfloor\log _{2} C\right\rfloor$;

(Surj): If $X(\mu)$ is finitely generated with $N$ generators, then for each $\varepsilon>0$ and each $D \in X(\mu)$ there is an $(1+\varepsilon) N\|D\|_{X(\mu) \text {-Lipschitz Alberti represen- }}$ tation $\mathcal{A}$ of a measure $\nu \ll \mu$ such that $D_{\mathcal{A}}=D$;

(WDens): In general the set of derivations of the form $D_{\mathcal{A}}$ is weak* dense in $X(\mu)$ in the sense that, given any $D \in X(\mu)$, for all $\varepsilon>0$, for each $\lambda \in L^{1}(\mu)$ and for each finite set of Lipschitz functions $\left\{g_{i}\right\}_{i=1}^{k}$ one can find an Alberti representation $\mathcal{A}$ of $\mu$ such that:

$$
\int_{X}|\lambda| \sum_{i=1}^{k}\left|D g_{i}-D_{\mathcal{A}} g_{i}\right| d \mu \leq \varepsilon .
$$

Recall that a sequence $\left\{f_{n}\right\} \subset \operatorname{Lip}_{\mathrm{b}}(X)$ converges to $f \in \operatorname{Lip}_{\mathrm{b}}(X)$ in the weak* topology $\left(f_{n} \stackrel{\mathrm{w}^{*}}{\longrightarrow} f\right)$ if $f_{n} \rightarrow f$ pointwise and $\sup _{n} \mathbf{L}\left(f_{n}\right)<\infty$. This result is a functional-analytic interpretation of constructions in [ACP05, ACP10, Bat12], that was obtained in [Sch14b, Sch13]; to obtain the optimal constants we were greatly helped by Andrea Marchese's PhD thesis.

Theorem 2.28 (Approximation Scheme). Let $K \subset X$ be compact and assume that $\mu \mathrm{L} K$ does not admit an Alberti representation with $f$-speed $\geq \delta$. Then there is 
a sequence $g_{k} \stackrel{\mathrm{w}^{*}}{\longrightarrow} f$ such that each $g_{k}$ is $\max (\mathbf{L}(f), \delta)$-Lipschitz and, for each $k$, there is an open set $U_{k} \supset K$ such that for each ball $B \subset U_{k}$ the restriction $g_{k} \mid B$ is $\delta$-Lipschitz.

This result [Sch14b, Sch13, Sec. 3.3] characterizes the local norm on the Weaver cotangent bundle.

Theorem 2.29 (Characterization of the local norm). Let $K \subset X$ be compact, $f: X \rightarrow \mathbb{R}$ Lipschitz and $\varepsilon>0$. Then $\mu\llcorner K$ admits a $(1,1+\varepsilon)$-biLipschitz Alberti representation with $f$-speed $\geq|d f|_{\mathcal{E}(\mu)}-\varepsilon$.

2.4. Differentiability spaces. We start with a brief review of differentiability spaces. For more details we refer to the original papers [Che99, Kei02, Kei04] or to the expository paper [KM11]. This structure has several names in the literature: (strong) measurable differentiabile structure, differentiable structure (in the sense of Cheeger and Keith), Lipschitz differentiability space, differentiability space. We highlight the features of differentiability spaces; contrary to some earlier papers, we do not assume a uniform bound on the dimension of the charts.

(1) There is a countable collection of charts $\left\{\left(U_{\alpha}, \phi_{\alpha}\right)\right\}_{\alpha}$, where $U_{\alpha} \subset X$ is Borel and $\phi_{\alpha}$ is Lipschitz, such that $X \backslash\left(\cup_{\alpha} U_{\alpha}\right)$ is $\mu$-null, and each real-valued Lipschitz function $f$ admits a first order Taylor expansion with respect to the components of $\phi_{\alpha}: X \rightarrow \mathbb{R}^{N_{\alpha}}$ at generic points of $U_{\alpha}$, i.e. there are (a.e. unique) measurable functions $\frac{\partial f}{\partial \phi_{\alpha}^{i}}$ on $U_{\alpha}$ such that:

$$
f(x)=f\left(x_{0}\right)+\sum_{i=1}^{N_{\alpha}} \frac{\partial f}{\partial \phi_{\alpha}^{i}}\left(x_{0}\right)\left(\phi_{\alpha}^{i}(x)-\phi_{\alpha}^{i}\left(x_{0}\right)\right)+o\left(d\left(x, x_{0}\right)\right)
$$

(for $\mu$-a.e. $x_{0} \in U_{\alpha}$ ).

The integer $N_{\alpha}$ is the dimension of the chart $\left\{\left(U_{\alpha}, \phi_{\alpha}\right)\right\}_{\alpha}$, and depends only on the set $U_{\alpha}$, not on the particular choice of the coordinate functions $\phi_{\alpha}$. If $\sup _{\alpha} N_{\alpha}<\infty$, it is called the differentiability or the analytic dimension. Since in this paper we are interested in the blow-ups of differentiability spaces, we can assume that there is a unique chart that covers all the space.

(2) There are measurable cotangent and tangent bundles $T^{*} X$ and $T X$; however, we will work with $\mathcal{X}(\mu)$ and $\mathcal{E}(\mu)$. By the result of [Sch13, Sch14b] $X(\mu)$ can be identified with the set of bounded measurable sections of $T X$ and $\mathcal{E}(\mu)$ with the set of bounded measurable sections of $T^{*} X$. Having locally trivialized $T^{*} X$ and $T X$, forms in $T^{*} X$ correspond to differentials of Lipschitz functions, and vectors in $T X$ give rise to examples of Weaver derivations in $\mathcal{X}(\mu)$.

Definition 2.31 (Variation and pointwise Lipschitz constants). For $f \in \operatorname{Lip}(X)$ we define the variation $\operatorname{Var} f(x, r)$ at $x$ at scale $r$, the big-Lip constant $\operatorname{Lip} f$ 
at $x$ and the small-lip constant $\operatorname{lip} f$ at $x$ as follows:

$$
\begin{aligned}
\operatorname{Var} f(x, r) & =\frac{1}{r} \sup _{y \in B(x, r)}|f(y)-f(x)| \\
\operatorname{Lip} f(x) & =\underset{r \searrow 0}{\limsup \operatorname{Var} f(x, r)} \\
\operatorname{lip} f(x) & =\liminf _{r \searrow 0} \operatorname{Var} f(x, r) .
\end{aligned}
$$

A metric measure space $(X, \mu)$ satisfies Keith's Lip-lip inequality with constant $K \geq 1$ if for each $f$ Lipschitz one has:

$$
\operatorname{Lip} f \leq K \operatorname{lip} f \quad \mu \text {-a.e. }
$$

Theorem 2.36 (Summary of results on differentiability spaces). This list summarizes relevant results on differentiability spaces:

(Cheeger): [Che99]; if $(X, \mu)$ is a doubling metric measure space which admits an abstract Poincaré inequality in the sense of Heinonen-Koskela [HK98] then $(X, \mu)$ is a differentiability space whose analytic dimension is bounded by an expression that depends only on the doubling constant $C_{\mu}$ of $\mu$ and the constants that appear in the Poincare inequality. Moreover, (2.35) holds with $K=1$;

(Keith): [Kei02, Kei04]; if $(X, \mu)$ is a doubling metric measure space which satisfies the Lip-lip inequality (2.35), then $(X, \mu)$ is a differentiability space whose analytic dimension is bounded by an expression that depends only on $C_{\mu}$ and $K$. Moreover [Kei03], the Poincaré inequality is stable under measured Gromov-Hausdorff convergence provided all the relevant constants are uniformly bounded; for example, blow-ups of PI-spaces are PI-spaces (with the same PI-exponent);

(Bate-Speight): [BS13]; if $(X, \mu)$ is a differentiability space then $\mu$ is asymptotically doubling in the sense that for $\mu$-a.e. $x$ there are $\left(C_{x}, r_{x}\right) \in(0, \infty)^{2}$ such that:

$$
\mu(B(x, 2 r)) \leq C_{x} \mu(B(x, r)) \quad\left(r \leq r_{x}\right) ;
$$

moreover, porous sets are $\mu$-null. In the following, to simplify the exposition, we will thus assume that differentiability spaces are doubling metric measure spaces.

(Bate): [Bat12, Bat15]; if $(X, \mu)$ is a differentiability space, $\mu$ admits many independent Alberti representations generalizing some of the results in $\mathbb{R}^{N}$ of $[\mathrm{ACP} 05, \mathrm{ACP} 10]$. Moreover, (2.35) holds where now $K$ is a Borel function that depends only on $X$;

(Schioppa): [Sch14b, Sch13]; in a differentiability space (2.35) always holds with $K=1$. Moreover, $(X, \mu)$ is a differentiability space if and only if for each Lipschitz function $f$ :

$$
\operatorname{Lip} f=|d f|_{\mathcal{E}(\mu)} \quad(\mu \text {-a.e. }),
$$

and (2.38) already encodes the condition that $\mu$ is asymptotically doubling. We will refer to (2.38) as the quantitative characterization of differentiability spaces. 
As a historical note, we point out that an earlier result (where one loses the optimal PI-exponent) on the stability of the Poincaré inequality under measured GromovHausdorff convergence can be found in [Che99, Sec.9].

2.5. The $p$-weak gradient. We recommend as a reference [ACM12] besides [AGS13].

Definition 2.39 (Absolutely continuous curves). Let $I$ be either a nondegenerate interval of $\mathbb{R}$ of the form $[a, b],(-\infty, a]$ or $[a, \infty)$, or the whole $\mathbb{R}$. A curve $\gamma: I \rightarrow X$ is absolutely continuous if there is a $g \in L^{1}\left(\mathcal{L}^{1} L \operatorname{dom} \gamma\right)$ such that for each $t, s \in \operatorname{dom} \gamma$ with $t \geq s$ one has:

$$
d(\gamma(t), \gamma(s)) \leq \int_{s}^{t} g(\tau) d \tau .
$$

Recall that if $\gamma: \operatorname{dom} \gamma \rightarrow X$ is absolutely continuous there is a minimal $g$ satisfying (2.40) which coincides $\mathcal{L}^{1}\llcorner\operatorname{dom} \gamma$-a.e. with the metric differential md $\gamma$ as defined in (2.21). Let $p \in[1, \infty)$ and $\gamma$ be an absolutely continuous curve; then $\gamma$ is of class $\mathrm{AC}^{p}$ if:

$$
\int_{\operatorname{dom} \gamma}(\operatorname{md} \gamma(t))^{p} d t<\infty
$$

The limit case $p=\infty$ corresponds to $\gamma$ being Lipschitz. The set of curves of class $\mathrm{AC}^{p}$ and with domain $[0,1]$ will be denoted by $\mathrm{AC}^{p}(X ;[0,1])$.

Definition 2.42 (Test plan). Let $(X, \mu)$ be a metric measure space and $p \in[1, \infty]$; a probability measure on $\mathrm{AC}^{p}(X ;[0,1])$ is called a $p$-test plan provided that:

$$
\begin{aligned}
\int_{\mathrm{AC}^{p}(X ;[0,1])} d \pi(\gamma) \int_{0}^{1}(\operatorname{md} \gamma(t))^{p} d t & <\infty \\
\operatorname{Ev}_{t \#} \pi & \leq C(\pi) \mu \quad(\forall t \in[0,1])
\end{aligned}
$$

for some constant $C(\pi)$, where $\mathrm{Ev}_{t}$ denotes the evaluation map:

$$
\begin{aligned}
\operatorname{Ev}_{t}: \operatorname{AC}^{p}(X ;[0,1]) & \rightarrow X \\
\gamma & \mapsto \gamma(t) .
\end{aligned}
$$

Definition 2.46 ( $p$-weak gradients). Let $(X, \mu)$ be a metric measure space, $f$ : $X \rightarrow \mathbb{R}$ and $g: X \rightarrow[0, \infty]$ Borel. Let $p \in(1, \infty)$ and $q$ denote the dual exponent $\frac{p}{p-1}$. The function $g$ is a $p$-weak upper gradient of $f$ if for each $q$-test plan $\pi$

$$
|f(\gamma(1))-f(\gamma(0))| \leq \int_{0}^{1} g \circ \gamma \operatorname{md} \gamma(t) d t
$$

holds $\pi$-a.s.

Assuming that $f$ has a $p$-weak upper gradient $g_{0}$ such that the measure $\mu \mathrm{L}\left\{g_{0}>\right.$ $0\}$ is $\sigma$-finite, then the set of $p$-weak upper gradients of $f$ contains a minimal element, the $p$-weak gradient of $f$, such that:

$$
|\nabla f|_{p, \mathrm{w}} \leq g \quad(\mu \text {-a.e. })
$$

for each $p$-weak upper gradient $g$ of $f$. 


\section{Blow-up of Weaver Derivations}

3.1. Variants of Gromov-Hausdorff convergence. We discuss some variants of Gromov-Hausdorff convergence (GH for short). Throughout this subsection metric spaces are assumed to be complete.

Definition 3.1 (GH-convergence). The GH-convergence of a sequence of pointed metric spaces $\left(X_{n}, p_{n}\right) \stackrel{\mathrm{GH}}{\longrightarrow}(Y, q)$ is equivalent to the existence of a pointed metric space $(Z, z)$, which we will call a container, and isometric embeddings:

$$
\begin{aligned}
& \iota_{n}: X_{n} \rightarrow Z \\
& \iota_{\infty}: Y \rightarrow Z
\end{aligned}
$$

such that:

(GH1): $\iota_{n}\left(p_{n}\right) \rightarrow \iota_{\infty}(q)$; one can even arrange $\iota_{n}\left(p_{n}\right)=\iota_{\infty}(q)=z$;

(GH2): for each $R>0$, one has:

$$
\begin{aligned}
& \lim _{n \rightarrow \infty} \sup _{y \in B(z, R) \cap \iota(Y)} \operatorname{dist}\left(\iota_{n}\left(X_{n}\right),\{y\}\right)=0, \\
& \lim _{n \rightarrow \infty} \sup _{y \in B(z, R) \cap \iota_{n}\left(X_{n}\right)} \operatorname{dist}(\iota(Y),\{y\})=0 .
\end{aligned}
$$

In the following we will often suppress $\iota_{n}$ and $\iota_{\infty}$ from the notation, just implying $X_{n}, Y \subset Z$. Note that each $y \in Y$ can be "approximated" by a sequence $x_{n} \in X_{n}$ such that $\iota_{n}\left(x_{n}\right) \rightarrow \iota_{\infty}(y)$ in $Z$. This notion is actually independent of the container $(Z, z)$, and one can represent each point $y \in Y$ by some sequence $x_{n} \in X_{n}$.

Definition 3.4 (mGH-convergence). We say that a pointed metric measure space $(X, \mu, p)$ is measure-normalized if $\int_{B(p, 1)}(1-d(p, x)) d \mu(x)=1$. The measured $\mathrm{GH}$-convergence ( $\mathrm{mGH}$ for short) of a sequence of measure-normalized pointed metric measure spaces $\left(X_{n}, \mu_{n}, p_{n}\right) \stackrel{\mathrm{mGH}}{\longrightarrow}(Y, \nu, q)$ is equivalent to requiring $\left(X_{n}, p_{n}\right) \stackrel{\mathrm{GH}}{\longrightarrow}$ $(Y, q)$, and that for each container $(Z, z)$ :

$$
\mu_{n} \stackrel{\mathrm{w}^{*}}{\longrightarrow} \nu \quad\left(\text { i.e. } \iota_{n, \#} \mu_{n} \stackrel{\mathrm{w}^{*}}{\longrightarrow} \iota_{\infty}, \# \nu\right) .
$$

Definition 3.6 (mfGH-convergence). A function space is a tuple $(X, \mu, p, \Phi)$ where $(X, \mu, p)$ is a normalized pointed metric measure space, and $\Phi$ is an at most countable collection of real-valued 1-Lipschitz functions on $X$ which vanish at $p$. We say that:

$$
\left(X_{n}, \mu_{n}, p_{n}, \Phi_{n}\right) \stackrel{\mathrm{mfGH}}{\longrightarrow}(Y, \nu, q, \Psi)
$$

in the mfGH-sense if:

$$
\text { (mfGH1): }\left(X_{n}, \mu_{n}, p_{n}\right) \stackrel{\mathrm{mGH}}{\longrightarrow}(Y, \nu, q) \text {; }
$$

(mfGH2): $\Phi_{n}$ and $\Psi$ have eventually the same cardinality;

(mfGH3): Let $\varphi_{n, k}$ denote the $k$-th element of $\Phi_{n}$ and $\psi_{k}$ the $k$-th element of $\Psi$; then whenever $x_{n}$ represents $y, \varphi_{n, k}\left(x_{n}\right) \rightarrow \psi_{k}(y)$.

Remark 3.8. Let $\left(X_{n}, \mu_{n}, p_{n}, \Phi_{n}\right) \stackrel{\mathrm{mfGH}}{\longrightarrow}(Y, \nu, q, \Psi)$ in the container $(Z, z)$. By replacing $Z$ with $Z \times \mathbb{R}$ and slightly shifting basepoints we may assume:

$$
\begin{aligned}
& X_{n} \cap X_{m}=\emptyset \quad(n \neq m) \\
& Y \cap X_{n}=\emptyset \quad(\forall n) .
\end{aligned}
$$


Then we might try to define $\varphi_{Z, k}=\varphi_{n, k}$ on $X_{n}$ and $\varphi_{Z, k}=\psi_{k}$ on $Y$. This yields a continuous function on $\bigcup_{n} X_{n} \cup Y$ which one might extend to $Z$. However, fix $R>0$; then $\varphi_{Z, k} \mid B(z, R)$ is almost 1-Lipschitz up to additive errors that depend on the Hausdorff-distance between $X_{n} \cap B(z, R)$ and $Y \cap B(z, R)$. By replacing $Z$ with $Z \times \mathbb{R}$, passing to a subsequence and shifting basepoints, one can verify the following lemma.

Lemma 3.10. Let $\left(X_{n}, \mu_{n}, p_{n}, \Phi_{n}\right) \stackrel{\mathrm{mfGH}}{\longrightarrow}(Y, \nu, q, \Psi)$ in the container $(Z, z)$. Up to passing to a subsequence and taking a new container of the form $(Z \times \mathbb{R},(z, 0))$, one can assume that for each $k \leq \# \Psi$ there is a 1-Lipschitz function $\varphi_{Z, k}: Z \rightarrow \mathbb{R}$ such that:

(1) $\varphi_{Z, k} \mid Y=\psi_{k}$;

(2) for each $R>0, k \leq \# \Psi$, there is an $N(R, k)$ such that, if $n \geq N(R, k)$, one has:

$$
\varphi_{Z, k} \mid B(z, R) \cap X_{n}=\varphi_{n, k} .
$$

For $\lambda>0$ and a metric space $X$, we denote by $\lambda X$ the metric space where the metric on $X$ has been rescaled by $\lambda$, i.e. $d_{\lambda X}=\lambda d_{X}$. Let $\Phi$ be a countable collection of 1-Lipschitz functions on $X$ and $p \in X$. Then we denote by $\Phi_{\lambda, p}$ the collection of 1-Lipschitz functions on $\lambda X$ given by:

$$
\Phi_{\lambda, p}=\{\lambda(\varphi-\varphi(p)): \varphi \in \Phi\} .
$$

Definition 3.13 (Blow-ups). A blow-up of $X$ at a point $p$ is a pointed metric space $(Y, q)$ such that for some sequence $\lambda_{n} \nearrow \infty$ :

$$
\left(X_{n}, p_{n}\right)=\left(\lambda_{n} X, p\right) \stackrel{\mathrm{GH}}{\longrightarrow}(Y, q) ;
$$

in this case we say that $(Y, q)$ is realized by the sequence of rescalings $\left\{\lambda_{n}\right\}$. The set of blow-ups of $X$ at $p$ will be denoted by $\operatorname{Bw}-u p(X, p)$.

Let $(X, \mu)$ be a metric measure space, $p \in X$ a basepoint and $\lambda>0$ a dilating factor; we define the normalization constant $c_{\mu}(p, \lambda)$ for the unit ball of $\lambda X$ centred at $p$ as follows:

$$
c_{\mu}(p, \lambda)=\left(\int_{B_{X}\left(p, \lambda^{-1}\right)}\left(1-\lambda d_{X}(p, x)\right) d \mu(x)\right)^{-1} .
$$

Note that in (3.15) we used the subscript $X$ to highlight that balls are taken with respect to the metric $d_{X}$ of $X$. A blow-up of $(X, \mu)$ at a point $p$ is a measurenormalized pointed metric space $(Y, \nu, q)$ such that for some sequence $\lambda_{n} \nearrow \infty$ :

$$
\left(X_{n}, \mu_{n}, p_{n}\right)=\left(\lambda_{n} X, c_{\mu}\left(p, \lambda_{n}\right) \mu, p\right) \stackrel{\mathrm{mGH}}{\longrightarrow}(Y, \nu, q) ;
$$

in this case we say that $(Y, \nu, q)$ is realized by the sequence of rescalings $\left\{\lambda_{n}\right\}$. The set of blow-ups of $X$ at $p$ will be denoted by $\operatorname{Bw}-u p(X, \mu, p)$.

A blow-up of $(X, \mu, \Phi)$ at a point $p$ is a function space $(Y, \nu, q, \Psi)$ such that for some sequence $\lambda_{n} \nearrow \infty$ :

$$
\left(X_{n}, \mu_{n}, p_{n}, \Phi_{n}\right)=\left(\lambda_{n} X, c_{\mu}\left(p, \lambda_{n}\right) \mu, p, \Phi_{\lambda_{n}, p}\right) \stackrel{\mathrm{mfGH}}{\longrightarrow}(Y, \nu, q, \Psi) ;
$$

in this case we say that $(Y, \nu, q, \Psi)$ is realized by the sequence of rescalings $\left\{\lambda_{n}\right\}$. The set of blow-ups of $X$ at $p$ will be denoted by $\operatorname{Bw}-u p(X, \mu, p, \Phi)$. 
The following theorem summarizes variants in the metric setting of Preiss' phenomenon that tangents of tangents are tangents [Pre87]; (1) is due to [LD11], (2) to [GMR15]; the proof of (3) is omitted as can be easily reconstructed from [GMR15]. It is clear that (3) can be generalized in further directions, e.g. in the context of blowing-up pseudodistances.

Theorem 3.18. (Shifted rescalings of blow-ups are blow-ups) Let $(X, \mu)$ be a doubling metric measure space and $\Phi$ a countable collection of 1-Lipschitz functions on $X$. Then for $\mu$-a.e. $p \in X$ the following holds:

(1) For each $(Y, q) \in \operatorname{Bw}-u p(X, p)$, for any $\left(\lambda, q^{\prime}\right) \in(0, \infty) \times Y$ one has $\left(\lambda Y, q^{\prime}\right) \in \operatorname{Bw}-u p(X, p)$; in particular, $\operatorname{Bw}-u p\left(Y, q^{\prime}\right) \subset \operatorname{Bw}-u p(X, p)$;

(2) For each $(Y, \nu, q) \in \operatorname{Bw}-u p(X, \mu, p)$, for any $\left(\lambda, q^{\prime}\right) \in(0, \infty) \times Y$ one has $\left(\lambda Y, c_{\nu}\left(q^{\prime}, \lambda\right) \nu, q^{\prime}\right) \in \operatorname{Bw}-u p(X, \mu, p) ;$ in particular, $\operatorname{Bw}-u p\left(Y, \nu, q^{\prime}\right) \subset$ $\mathrm{Bw}-\operatorname{up}(X, \mu, p)$;

(3) For each $(Y, \nu, q, \Psi) \in \operatorname{Bw}-u p(X, \mu, p, \Phi)$, for any $\left(\lambda, q^{\prime}\right) \in(0, \infty) \times Y$ one has $\left.\left(\lambda Y, c_{\nu}\left(q^{\prime}, \lambda\right) \nu, q^{\prime}\right), q^{\prime}, \Psi_{\lambda, q^{\prime}}\right) \in \operatorname{Bw}-u p(X, \mu, p, \Phi) ;$ in particular, $\mathrm{Bw}-\mathrm{up}\left(Y, \nu, q^{\prime}, \Psi\right) \subset \mathrm{Bw}-u p(X, \mu, p, \Phi)$;

3.2. Blow-up of Weaver derivations and weak convergence for normal currents. In this subsection we analyze how rescalings affect the modules $X(\mu)$ and $\mathcal{E}(\mu)$. When we want to highlight an object that refers to the rescaled space $\lambda X$ (resp. the original space $X$ ), we add $\lambda X$ (resp. $X$ ) to the notations. Recall that in this paper we use the notation $\mathbf{L}(f)$ for the global Lipschitz constant of $f$. We then sketch the details of how to use a result in [CKS15, Sec. 7] to blow-up Weaver derivations. The following lemma is elementary and the proof is omitted.

Lemma 3.19. Let $X$ be a metric space, $\lambda>0$ and $f$ a real-valued Lipschitz function defined on $X$. Then $\mathbf{L}_{X}(f)=C$ if and only if $\mathbf{L}_{\lambda X}(\lambda f)=C$, and $\mathbf{L}_{X}\left(\lambda^{-1} f\right)=C$ if and only if $\mathbf{L}_{\lambda X}(f)=C$.

Definition 3.20 (Rescaling of an Alberti representation). Let $\mathcal{A}=[Q, w]$ be an Alberti representation of the measure $\mu$ on $X$; let $\lambda>0$ and define $\operatorname{Res}_{\lambda}: \operatorname{Frag}(X) \rightarrow$ $\operatorname{Frag}(\lambda X)$ as follows:

$$
\begin{aligned}
\operatorname{dom}\left(\operatorname{Res}_{\lambda}(\gamma)\right) & =\lambda \operatorname{dom} \gamma \\
\operatorname{Res}_{\lambda}(\gamma)(t) & =\gamma(t / \lambda) .
\end{aligned}
$$

Let $p \in X$ and define $\operatorname{Res}_{\lambda, p}: \operatorname{Frag}(X) \rightarrow \operatorname{Frag}(\lambda X)$ as follows:

$$
\begin{aligned}
\operatorname{dom}\left(\operatorname{Res}_{\lambda, p}(\gamma)\right) & =\lambda\left(\operatorname{dom} \gamma-s_{\gamma, p}\right) \\
\operatorname{Res}_{\lambda, p}(\gamma)(t) & =\gamma\left(t / \lambda+s_{\gamma, p}\right),
\end{aligned}
$$

where $s_{\gamma, p}$ is such that:

$$
d\left(\gamma\left(s_{\gamma, p}\right), p\right)=\min _{t \in \operatorname{dom} \gamma} d(\gamma(t), p)
$$

Note that a measurable choice of $\operatorname{Res}_{\lambda, p}$ can be obtained via a measurable selection principle. The rescalings $\mathcal{A}_{\lambda}$ and $\mathcal{A}_{\lambda, p}$ are defined as follows:

$$
\begin{aligned}
\mathcal{A}_{\lambda} & =\left[\operatorname{Res}_{\lambda \#} Q, w\right] \\
\mathcal{A}_{\lambda, p} & =\left[\operatorname{Res}_{\lambda, p} Q, w\right] .
\end{aligned}
$$

Note that if $\mathcal{A}$ is $\left[C_{0}, D_{0}\right]$-biLipschitz on $X$, then $\mathcal{A}_{\lambda}$ and $\mathcal{A}_{\lambda, p}$ are $\left[C_{0}, D_{0}\right]$-biLipschitz on $\lambda X$. 
Lemma 3.25. Let $f \in \operatorname{Lip}_{\mathrm{b}}(X)$; then

$$
\lambda^{-1}\left|d^{(X)} f\right|_{\varepsilon(\mu ; X)}=\left|d^{(\lambda X)} f\right|_{\mathcal{E}(\mu ; \lambda X)} .
$$

Proof. Assume that $\left|d^{(X)} f\right|_{\mathcal{E}(\mu ; X)} \geq \alpha$ on a set $S$. Fix $\varepsilon>0$; then by Theorem 2.29 one can find a $(1,1+\varepsilon)$-biLipschitz Alberti representation $\mathcal{A}_{S}$ of $\mu \mathrm{L} S$ with $f$-speed $\geq \alpha-\varepsilon$. This means that for $Q$-a.e. $\gamma$ and $\mathcal{L}^{1}\llcorner$ dom $\gamma$-a.e. $t$ one has:

$$
(f \circ \gamma)^{\prime}(t) \geq(\alpha-\varepsilon) \text {. }
$$

Now the rescaled representation $\mathcal{A}_{S, \lambda}$ gives a $(1,1+\varepsilon)$-biLipschitz (wrt. the metric on $\lambda X)$ Alberti-representation of $\mu\llcorner S$ with a lower bound on the $f$-speed, that can be obtained using:

$$
\begin{aligned}
\left(f \circ \operatorname{Res}_{\lambda}(\gamma)\right)^{\prime}(t) & =\frac{d}{d t}(f(\gamma(t / \lambda))) \\
& =\lambda^{-1}(f \circ \gamma)^{\prime}(t / \lambda) \geq \lambda^{-1}(\alpha-\varepsilon) \\
& \geq \lambda^{-1}(1+\varepsilon)^{-1}(\alpha-\varepsilon) \operatorname{md}^{\operatorname{Res}_{\lambda}}(\gamma)(t),
\end{aligned}
$$

where the metric differential $\operatorname{Res}_{\lambda}(\gamma)(t)$ is computed with respect to the metric on $\lambda X$. Thus:

$$
\left|d^{(\lambda X)} f\right|_{\varepsilon(\mu ; \lambda X)} \geq \lambda^{-1} \alpha \quad \mu \text {-a.e. on } S \text {. }
$$

Theorem 3.30 (Blow-up of Weaver derivations). Let $(X, \mu)$ be a complete separable doubling metric measure space where $\mu$ is an asymptotically doubling Radon measure. Let $\Phi$ be a countable collection of real-valued 1-Lipschitz functions on $X$. Let $D \in X(\mu ; X)$ be of the form $D=D_{\mathcal{A}}$ where $\mathcal{A}$ is a $\left[C_{0}, D_{0}\right]$-biLipschitz Alberti representation of $\mu$. Then there is a $\mu$-full measure Borel set $X_{\text {blow }}$ such that for each $p \in X_{\text {blow }}$ and each $(Y, \nu, q, \Psi) \in \operatorname{Bw}-u p(X, \mu, p, \Phi)$ one can blow-up $D$ as follows. Assume that $(Y, \nu, q, \Psi)$ is realized by some sequence of rescalings $\left\{\lambda_{n}\right\}_{n}$ and let $\mathcal{A}_{n}=\mathcal{A}_{\lambda_{n}, p}$ and $D^{(n)}=D_{\mathcal{A}_{n}}$ be the corresponding derivation in $X\left(\mu_{n} ; X_{n}\right)$. Then there is an Alberti representation $\mathcal{A}_{\infty}=\left[Q_{\infty}, 1\right]$ of $\nu$ such that, defining $D^{(\infty)}=D_{\mathcal{A}_{\infty}}$, the following holds:

(Bw-up1): $\operatorname{spt} Q_{\infty}$ consists of lines in $Y$ with constant speed in $\left[C_{0}, D_{0}\right]$;

(Bw-up2): For each $\varphi \in \Phi$ and $\gamma$ in $\operatorname{spt} Q_{\infty}$ there is a $c_{\varphi, \gamma} \in[-1,1]$ such that, if $\psi \in \Psi$ denotes the corresponding blow-up of $\varphi$ :

$$
(\psi \circ \gamma)^{\prime}(t)=c_{\varphi, \gamma} \operatorname{md} \gamma(t) \quad(\forall t \in \mathbb{R}) ;
$$

(Bw-up3): Suppose that $\left(X_{n}, \mu_{n}, x_{n}, \Phi_{\lambda_{n}, x}\right) \stackrel{\mathrm{mfGH}}{\longrightarrow}(Y, \nu, y, \Psi)$ in the container $(Z, z)$; then up to passing to a subsequence (which might depend on the container) one can assume that for each $(g, f) \in C_{c}(Z) \times \operatorname{Lip}_{\mathrm{b}}(Z)$ one has:

$$
\lim _{n \rightarrow \infty} \int g D^{(n)} f d \mu_{n}=\int g D^{(\infty)} f d \nu .
$$

Remark 3.33. The statement (3.32) has a cleaner interpretation in the language of metric currents [Sch14a]: the metric currents:

$$
T_{n}(g, f)=\int g D^{(n)} f d \mu_{n}
$$


are converging to the normal current:

$$
T(g, f)=\int g D^{(\infty)} f d \nu
$$

in the weak topology.

Remark 3.36. One can also remove from Theorem 3.30 the assumption that $X$ is doubling. In that case one has to replace $X$ by an appropriate full-measure Borel $\tilde{X} \subset X$ and blow-up $\tilde{X}$. However, note that if porous sets are not $\mu$-null, there will be a set of positive measure where $\tilde{X}$ and $X$ do not have the same blow-ups (e.g. it might happen that one cannot apply Gromov's compactness Theorem to $X)$. However, if one uses ultrafilters, one can show that if $(Y, y)$ is a blow-up of $X$ at $x \in \tilde{X}$, then there is a blow-up $(\tilde{Y}, \nu, y)$ of $(\tilde{X}, \mu\llcorner\tilde{X})$ at $x$ such that $\tilde{Y} \subset Y$.

Proof. The proof can be reconstructed from the argument in [CKS15, Sec. 7] where the result is stated in a less general context: $(X, \mu)$ is a differentiability space and $\Phi$ is a finite set of Lipschitz functions. The only item that requires further justification is (Bw-up3). We highlight the additional arguments; we will refer to the notation and setting in [CKS15, Sec. 7]; in particular, $r_{n}=\lambda_{n}^{-1}$ and for $\gamma \in \operatorname{Frag}(X)$ we define:

$$
\Psi(\gamma)=\gamma_{\sharp}\left(\mathcal{L}^{1}\llcorner\operatorname{dom} \gamma) .\right.
$$

However note that we are using a convention to normalize the measures different from that in [CKS15], where measures are rescaled to give unit mass to the open unit ball. This essentially amounts to replacing some terms in [CKS15, Sec. 7] of the form $\mu^{\prime}(B(x, r))$ with $1 / c_{\mu^{\prime}}\left(x, r^{-1}\right)$, see the following discussion for more details. In [CKS15, Sec. 7] we have performed some preliminary steps:

(PS1): If $\mathcal{A}=[Q, w]$, instead of working with $\mu$, we work with the measure $\mu^{\prime}$ corresponding to the Alberti representation $\mathcal{A}=[Q, 1]$. This can be done as $\mu$ is asymptotically doubling and as $D_{\mathcal{A}}$ is not affected on the set where $w \neq 0$;

(PS2): For $\gamma \in \operatorname{spt} Q$ we have split $\Psi(\gamma)$ in a regular part $\Psi_{\mathrm{PAR}(\varepsilon, S)}(\gamma)$ and an irregular part $\Psi_{\operatorname{PAR}(\varepsilon, S)}^{c}(\gamma)$ (compare equation (7.34) in [CKS15, Sec. 7]). There are straightforward modifications for the regularity requirements to handle a countable collection $\Phi$; here $\varepsilon$ and $S$ play the rôle of parameters selecting $(S)$ how long $\gamma$ is, and $(\varepsilon)$ how close $\gamma$ is to a constant speed segment on which the first $\lfloor S\rfloor$ elements of $\Phi$ are close to affine maps;

(PS3): We have chosen an $l^{1}$-sequence $\left\{\varepsilon_{m}\right\} \subset(0, \infty)$ and used (Lemma 7.35 in [CKS15, Sec. 7]) measure-differentiation to find a Borel set $U$ and scales $s_{m} \leq S_{m}$ such that:

$$
\begin{aligned}
\mu(X \backslash U) & \leq \sum_{m=1}^{\infty} \varepsilon_{m} \\
\mu_{\mathrm{PAR}\left(\varepsilon_{m}, S_{m}\right)}^{\prime c}(B(x, r)) & \leq \frac{\varepsilon_{m}}{c_{\mu^{\prime}}\left(x, r^{-1}\right)} \quad\left(\forall x \in U, r \leq s_{m}\right),
\end{aligned}
$$

where

$$
\begin{aligned}
& \mu_{\mathrm{PAR}\left(\varepsilon_{m}, S_{m}\right)}^{\prime}=\int \Psi_{\mathrm{PAR}\left(\varepsilon_{m}, S_{m}\right)}(\gamma) d Q(\gamma) \\
& \mu_{\mathrm{PAR}\left(\varepsilon_{m}, S_{m}\right)}^{\prime c}=\int \Psi_{\mathrm{PAR}\left(\varepsilon_{m}, S_{m}\right)}^{c}(\gamma) d Q(\gamma) .
\end{aligned}
$$


Note that the second equation in (3.38) differs from the corresponding one in Lemma 7.35 of [CKS15, Sec. 7], as we have replaced $\mu^{\prime}(B(x, r))$ with $1 / c_{\mu^{\prime}}\left(x, r^{-1}\right)$. This is possible as $\mu^{\prime}$ is asymptotically doubling and the function $1-r^{-1} d(\cdot, x)$ has value at least $\frac{1}{2}$ on $B(x, r / 2)$; this also implies that for each fixed $R_{0} \geq 1$ one can find $C\left(R_{0}\right)$ such that $c_{\mu^{\prime}}\left(x, r^{-1}\right) \lesssim_{C\left(R_{0}\right)}$ $c_{\mu^{\prime}}\left(x, R_{0}^{-1} r^{-1}\right)$ : we will not insist on this point any further;

(PS4): We take $x \in U$ and, up to enlarging $(Z, z)$, we assume that $(Z, z)$ is a Banach space.

We now fix $R_{0}$ large enough so that, given $(g, f) \in C_{c}(Z) \times \operatorname{Lip}_{\mathrm{b}}(Z)$, spt $g \subset$ $B\left(z, R_{0}\right)$. As $\left|D_{\mathcal{A}_{n}} f\right| \leq D_{0} \mathbf{L}(f)$, we conclude from equation (7.55) in [CKS15, Sec. 7] that:

$$
\begin{aligned}
\left|c_{\mu^{\prime}}\left(p, r_{n}^{-1}\right) \int_{B_{X_{n}}\left(p, R_{0}\right)} g D_{\mathcal{A}_{n}} f d \mu_{\mathrm{PAR}\left(\varepsilon_{m}, S_{m}\right)}^{\prime c}\right| & \leq\|g\|_{\infty} D_{0} \mathbf{L}(f) c_{\mu^{\prime}}\left(p, r_{n}^{-1}\right) \\
& \times \mu_{\mathrm{PAR}\left(\varepsilon_{m}, S_{m}\right)}^{\prime c}\left(B_{X}\left(p, r_{n} R_{0}\right)\right) ;
\end{aligned}
$$

note that we are regarding $\mu_{\mathrm{PAR}\left(\varepsilon_{m}, S_{m}\right)}^{\prime c}$ as a measure on $X_{n}$ and are suppressing from the notation the isometric embeddings in $Z$.

As $Z$ is a Banach space, one can introduce a "filling map" Fill which fills fragments to curves (details are discussed in [CKS15, Sec. 7]). Using the Hausdorff topology on fragments one can introduce reparametrization maps $\left\{\operatorname{Rep}_{n}\right\}_{n}$ which agree, up possibly to a translation, with the map $\left\{\operatorname{Res}_{\lambda_{n}, x}\right\}_{n}$.

Let $\Gamma_{X_{n}} \subset \operatorname{Frag}(X)$ denote the set of those $\left[C_{0}, D_{0}\right]$-biLipschitz fragments which intersect $\bar{B}_{X}\left(x, 2 R_{0} r_{n}\right)$, and by $\tilde{\Gamma}_{X_{n}} \subset \Gamma_{X_{n}}$ the Borel subset of those $\gamma$ such that:

$$
\chi_{B_{X_{n}}\left(x, R_{0}\right)} \Psi_{\operatorname{PAR}\left(\varepsilon_{m}, S_{m} / r_{n}\right)}\left(\operatorname{Rep}_{n}(\gamma)\right) \neq 0 .
$$

Then combining equations (7.60) and (7.66) in [CKS15, Sec. 7] with the definition of $D^{(n)}$ one arrives at the estimate:

$$
\begin{aligned}
\mid \int_{X_{n}} g D^{(n)} f d \mu_{n}^{\prime} & -c_{\mu^{\prime}}\left(p, r_{n}^{-1}\right) \int_{\tilde{\Gamma}_{X_{n}}} d Q(\gamma) \int r_{n} g \circ\left(\text { Fill } \circ \operatorname{Rep}_{n}\right)(\gamma) \\
\times & \left(f \circ\left(\text { Fill } \circ \operatorname{Rep}_{n}\right)(\gamma)\right)^{\prime} \chi_{B_{X_{n}}\left(x, R_{0}\right)} d \Psi\left(\text { Fill } \circ \operatorname{Rep}_{n}(\gamma)\right) \mid \\
\leq & \|g\|_{\infty} D_{0} \mathbf{L}(f)\left(O\left(\varepsilon_{m}\right)+C\left(C_{0}, D_{0}\right) \varepsilon_{m}\right. \\
& \left.\times c_{\mu^{\prime}}\left(p, r_{n}^{-1}\right) \mu^{\prime}\left(B_{X}\left(p, 2 r_{n} R_{0}\right)\right)\right) .
\end{aligned}
$$

Note that by using the property of $\mu^{\prime}$ being asymptotically doubling one can find a uniform bound on $c_{\mu^{\prime}}\left(p, r_{n}^{-1}\right) \mu^{\prime}\left(B_{X}\left(p, 2 r_{n} R_{0}\right)\right)$ which depends on $R_{0}$ but not on $n$. Letting:

$$
Q_{n}=c_{\mu^{\prime}}\left(p, r_{n}^{-1}\right) r_{n} \text { Fill } \circ \operatorname{Rep}_{n}(\gamma)_{\sharp} Q\left\llcorner\tilde{\Gamma}_{X_{n}}\right.
$$

one can use the mass estimate (equation (7.71) in [CKS15, Sec. 7]) to show that $Q_{n} \stackrel{\mathrm{w}^{*}}{\longrightarrow} Q_{R_{0}}$ by passing to a subsequence. By a diagonal argument (compare the proof of Lemma 7.78 in [CKS15, Sec. 7]) which involves $R_{0} \nearrow \infty$ one can obtain a limit $Q_{\infty}$ of the $Q_{R_{0}}$ and an Alberti representation $\mathcal{A}_{\infty}=\left[Q_{\infty}, 1\right]$. To prove (3.32) it suffices to use the definition of the weak* topology for Radon measures by showing 
that if $\Omega$ is a closed subset of $\operatorname{Curves}(Z)$ with all elements of $\Omega$ having their domain contained in a given bounded interval of $\mathbb{R}$, and with $\sup _{\Omega \ni \gamma} \mathbf{L}(\gamma)<\infty$, then the map:

$$
\Omega \ni \gamma \mapsto \int g \circ \gamma(f \circ \gamma)^{\prime} d \Psi(\gamma)
$$

is continuous. This reduces to the weak* continuity of the derivation $\partial_{x} \in X\left(\mathcal{L}^{1}\right)$ on the real line [Wea00].

Corollary 3.45. Let $(X, \mu)$ be a metric measure space with $\mu$ asymptotically doubling and assume that $\mathcal{E}(\mu)$ is free on $\left\{d \varphi_{i}\right\}_{i=1}^{N}$ where each $\varphi_{i}$ is 1-Lipschitz, and let $\Phi=\left\{\varphi_{i}\right\}_{i=1}^{N}$. Then for $\mu$-a.e. $x$ and for each $(Y, \nu, y, \Psi) \in \operatorname{Bw}-u p(X, \mu, x, \Phi)$ the following holds:

(FormBlow1): The submodule of $\mathcal{E}(\nu)$ generated by the $\left\{d \psi_{i}\right\}_{i=1}^{N}$ (where $\left.\left\{\psi_{i}\right\}_{i=1}^{N}=\Psi\right)$ is free;

(FormBlow2): For each $a \in \mathbb{R}^{N}$ there is an Alberti representation $\left[Q_{a}, 1\right]$ of $\nu$, where $Q_{a}$ is concentrated on the set of unit-speed lines of $Y$ satisfying:

$$
\sum_{i=1}^{N} a_{i}\left(\psi_{i} \circ \gamma(t)-\psi_{i} \circ \gamma(s)\right)=\left|\sum_{i=1}^{N} a_{i} d \varphi_{i}(x)\right|_{\varepsilon(\mu)}(t-s) \quad(\forall t \geq s) ;
$$

(FormBlow3): Moreover, if $(X, \mu)$ is a differentiability space, one also has:

$$
\left|\sum_{i=1}^{N} a_{i} d \varphi_{i}(x)\right|_{\mathcal{E}(\mu)}=\operatorname{Lip}\left(\sum_{i=1}^{N} a_{i} \varphi_{i}\right)(x)=\mathbf{L}\left(\sum_{i=1}^{N} a_{i} \psi_{i}\right) .
$$

Proof. Step1: Blowing-up a single function $f$.

Assume that $f$ is 1-Lipschitz with $d f \neq 0 \mu$-a.e.; by Theorem 2.29, for each $n, \mu$ admits a $\left(1,1+\frac{1}{n}\right)$-biLipschitz Alberti representation $\mathcal{A}_{n}$ with $f$ speed $\geq$ $|d f|_{\mathcal{E}(\mu)}-1 / n$; note also that $|d f|_{\mathcal{E}(\mu)}$ is necessarily an upper bound on the $f$-speed of any Alberti representation. We use Theorem 3.30 to find a $\mu$-full measure subset $U$ where one can blow-up each $\mathcal{A}_{n}$ and on which $|d f|_{\mathcal{E}(\mu)}$ is approximately continuous. For a Lebesgue point $x$ of $U$ let $(Y, \nu, y,\{g\}) \in \mathrm{Bw}-u p(X, \mu, x,\{f\})$, and denote by $\mathcal{A}_{n, \infty}=\left[Q_{n}, 1\right]$ the corresponding blow-up of $\mathcal{A}_{n}$. Then $Q_{n}$ is concentrated on the set of lines in $Y$ with constant speed in $[1,1+1 / n]$ and which satisfy:

$$
\begin{array}{r}
g(\gamma(t))-g(\gamma(s)) \in\left[\left(|d f(x)|_{\mathcal{E}(\mu)}-\frac{1}{n}\right)(t-s),\left(1+\frac{1}{n}\right)|d f(x)|_{\mathcal{E}(\mu)}(t-s)\right] \\
(\forall t \geq s) .
\end{array}
$$

By a compactness argument one obtains an Alberti representation $\mathcal{A}_{\infty}=\left[Q_{\infty}, 1\right]$ of $\nu$ where $Q_{\infty}$ is concentrated on the set of unit speed lines of $Y$ satisfying:

$$
g(\gamma(t))-g(\gamma(s))=|d f(x)|_{\mathcal{E}(\mu)}(t-s) \quad(\forall t \geq s) .
$$

Step 2: Approximation by rational combinations.

We apply Step 1 to each function $\Phi_{\mathbb{Q}}=\left\{\sum_{i=1}^{N} a_{i} \varphi_{i}\right\}_{a \in \mathbb{Q}^{N}}$, and one also assumes that $x$ is an approximate continuity point of each map:

$$
\tilde{x} \mapsto\left|\sum_{i=1}^{N} a_{i} d \varphi_{i}(\tilde{x})\right|_{\mathcal{E}(\mu)} \quad\left(a \in \mathbb{Q}^{N}\right) .
$$


To obtain (FBlow1), (FBlow2) one then uses the fact that $\mathbb{Q}^{N}$ is dense in $\mathbb{R}^{N}$ and the linearity of blow-ups, i.e. that the blow-up of $\sum_{i=1}^{N} a_{i} \varphi_{i}$ is $\sum_{i=1}^{N} a_{i} \psi_{i}$. (FBlow3) follows from the quantitative characterization of differentiability spaces ((Schioppa) in Theorem 2.36) and from the fact that given a Lipschtiz function $f$, at $\mu$-a.e. $x$ any blow-up of $f$ at $x$ has Lipschitz constant at most $\operatorname{Lip} f(x)$.

\section{A LOCAL APPROACH TO FAIL DifFERENTIABILITY}

In this section we discuss a methodology for the gluing part of the argument. Even though we end up using "cubical" tiles in Section 5, other geometries for tiles might be helpful in deducing other properties of blow-ups. The following theorem summarizes sufficient conditions to show that a metric measure space is not a differentiability space; we follow an approach motivated by Weaver derivations; for the proof see [Sch13, Sec. 5.3] (or [Bat15] for an approach via charts).

Theorem 4.1. Let $(X, \mu)$ be a metric measure space with $\mu(X)=1$. Assume that for some $\left(\alpha_{\mathrm{var}}, L\right) \in(0, \infty)^{2}$ for each $\varepsilon>0$ there is an L-Lipschitz function $f_{\varepsilon}$ such that:

(SmDiff): $\mu\left(\left\{x \in X:\left|d f_{\varepsilon}(x)\right|_{\mathcal{E}(\mu)}>\varepsilon\right\}\right) \leq \varepsilon ;$

(PosVar): There is a Borel set $X_{\mathrm{var}} \subset X$ with $\mu\left(X \backslash X_{\mathrm{var}}\right) \leq \varepsilon$, and such that for each $x \in X_{\mathrm{var}}$ there is an $x_{\mathrm{var}}=x_{\mathrm{var}}(x)$ satisfying:

$$
\begin{aligned}
d\left(x, x_{\mathrm{var}}\right) & \in(0, \varepsilon] \\
\left|f_{\varepsilon}(x)-f_{\varepsilon}\left(x_{\mathrm{var}}\right)\right| & \geq \alpha_{\mathrm{var}} d\left(x, x_{\mathrm{var}}\right) .
\end{aligned}
$$

Then $(X, \mu)$ is not a differentiability space.

Definition 4.4 (Tiles). Let $\left(L, \alpha_{\mathrm{var}}, \varepsilon_{\mathrm{grad}}, \varepsilon_{\mathrm{loss}}, r\right) \in(0, \infty)^{5}$; an $\left[L, \alpha_{\mathrm{var}}, \varepsilon_{\mathrm{grad}}, \varepsilon_{\mathrm{loss}}, r\right]$ tile at $x$ is a pair $\left(S_{x}, f_{x}\right)$ such that:

(T1): $S_{x}$ is closed, $S_{x} \subset B(x, r)$ and $\operatorname{diam} S_{x} \approx_{L} r$;

(T2): $\mu\left(S_{x}\right) \gtrsim_{L} \mu(B(x, r))$;

(T3): $f_{x}$ is $L$-Lipschitz and for each $p \in S_{x}$ one has:

$$
\left|f_{x}(p)\right| \leq L d\left(p, S_{x}^{c}\right)
$$

(T4): $\mu\left(\left\{p \in S_{x}:\left|d f_{x}(p)\right|_{\mathcal{E}(\mu)}>\varepsilon_{\text {grad }}\right\}\right) \leq \varepsilon_{\text {loss }} \mu\left(S_{x}\right) ;$

(T5): There is a Borel set $S_{x, \text { var }} \subset S_{x}$ such that $\mu\left(S_{x} \backslash S_{x, \text { var }}\right) \leq \varepsilon_{\operatorname{loss}} \mu\left(S_{x}\right)$ and for each $y \in S_{x, \mathrm{var}}$ there is a $y_{\mathrm{var}}=y_{\mathrm{var}}(y)$ such that:

$$
\begin{aligned}
d\left(y, y_{\mathrm{var}}\right) & \in\left(0, \varepsilon_{\text {loss }}\right] \\
\left|f_{x}(y)-f_{x}\left(y_{\mathrm{var}}\right)\right| & \geq \alpha_{\mathrm{var}} d\left(y, y_{\mathrm{var}}\right) .
\end{aligned}
$$

Theorem 4.8. Let $(X, \mu)$ be a complete metric measure space with $\mu(X)=1$, and such that the Vitali Covering Lemma holds for $\mu$. Assume that there are constants $\left(L, \alpha_{\mathrm{var}}, \varepsilon_{\mathrm{grad}}, \varepsilon_{\mathrm{loss}}\right) \in(0, \infty)^{4}$ such that for $\mu$-a.e. $x \in X$ there is a sequence of scales $\left\{r_{n}=r_{n}(x) \searrow 0\right\}_{n}$ such that for each $n$ there is an $\left[L, \alpha_{\mathrm{var}}, \varepsilon_{\text {grad }}, \varepsilon_{\mathrm{loss}}, r_{n}\right]$ tile at $x$. Then the assumption of Theorem 4.1 holds with $L$ replaced by $2 L$, with $\alpha_{\mathrm{var}}$ replaced by $\alpha_{\mathrm{var}} / 2$, and with $\varepsilon=\max \left(\varepsilon_{\mathrm{grad}}, \varepsilon_{\mathrm{loss}}\right)$.

Proof. By (T1), (T2) tiles are closed and comparable to balls in measure and shape; thus by the Vitali Covering Lemma we can find tiles $\left\{\left(S_{x_{i}}, f_{x_{i}}\right)\right\}_{i}$ such that the sets $\left\{S_{x_{i}}\right\}$ are pairwise disjoint and $\mu\left(X \backslash \bigcup_{i} S_{x_{i}}\right)=0$. We let $f=f_{x_{i}}$ on each $S_{x_{i}}$ and $f=0$ on $X \backslash \bigcup_{i} S_{x_{i}}$. 
Step 1: $f$ is 2L-Lipschitz.

We will use (T3) to compare values of $f$ at points belonging to different tiles. If $x, y \in S_{x_{i}}$ we have:

$$
|f(x)-f(y)|=\left|f_{x_{i}}(x)-f_{x_{i}}(y)\right| \leq L d(x, y)
$$

because $f_{x_{i}}$ is $L$-Lipschitz. If $x \in S_{x_{i}}$ and $y \in S_{x_{j}}$ for $i \neq j$ :

$$
\begin{aligned}
|f(x)-f(y)| & \leq\left|f_{x_{i}}(x)\right|+\left|f_{x_{j}}(y)\right| \leq L\left[d\left(x, S_{x_{i}}^{c}\right)+d\left(y, S_{x_{j}}^{c}\right)\right] \\
& \leq 2 L d(x, y) .
\end{aligned}
$$

If $x \in S_{x_{i}}$ and $y \in X \backslash \bigcup_{i} S_{x_{i}}$ we have:

$$
|f(x)-f(y)|=\left|f_{x_{i}}(x)\right| \leq L d\left(x, S_{x_{i}}^{c}\right) \leq L d(x, y) .
$$

If $x, y \in X \backslash \bigcup_{i} S_{x_{i}}$ :

$$
|f(x)-f(y)|=0 .
$$

Step 2: (SmDiff) holds with $\varepsilon=\max \left\{\varepsilon_{\text {grad }}, \varepsilon_{\text {loss }}\right\}$.

We will use (T4) on each tile. The exterior differential $d$ is a local operator [Wea00], i.e.:

$$
d f=d f_{x_{i}} \quad \mu\left\llcorner S_{x_{i}}\right. \text {-a.e. }
$$

Thus:

$$
\begin{aligned}
\mu\left(\left\{x \in X:|d f(x)|_{\varepsilon(\mu)}>\varepsilon\right\}\right) & \leq \sum_{i} \mu\left(\left\{x \in S_{x_{i}}:\left|d f_{x_{i}}(x)\right|_{\mathcal{E}(\mu)}>\varepsilon_{\text {grad }}\right\}\right) \\
& \leq \sum_{i} \varepsilon_{\text {loss }} \mu\left(S_{x_{i}}\right) \\
& \leq \varepsilon
\end{aligned}
$$

Step 3: (PosVar) holds with $\alpha_{\text {var }} / 2$ replacing $\alpha_{\text {var }}$.

We will use (T5) and the fact that $f$ and $f_{x_{i}}$ vanish on the boundary of each tile. Let $X_{\mathrm{var}}=\bigcup_{i} S_{x_{i} \text {,var }}$ so that $\mu\left(X \backslash X_{\mathrm{var}}\right) \leq \varepsilon$. Let $y \in S_{x_{i} \text {,var }}$ and assume that the $y_{\text {var }}$ corresponding to $f_{x_{i}}$ and $y$ is in $S_{x_{i}}$. Then:

$$
\left|f(y)-f\left(y_{\mathrm{var}}\right)\right| \geq\left|f_{x_{i}}(y)-f_{x_{i}}\left(y_{\mathrm{var}}\right)\right| \geq \alpha_{\mathrm{var}} d\left(y, y_{\mathrm{var}}\right) .
$$

If $y_{\text {var }}$ does not lie in $S_{x_{i}}$ we conclude that:

$$
|f(y)|=\left|f_{x_{i}}(y)-f_{x_{i}}\left(y_{\mathrm{var}}\right)\right| \geq \alpha_{\mathrm{var}} d\left(y, y_{\mathrm{var}}\right) \geq \alpha_{\mathrm{var}} d\left(y, S_{x_{i}}^{c}\right) .
$$

We then choose $\tilde{y} \in \partial S_{x_{i}}$ such that:

$$
d(y, \tilde{y}) \leq \min \left(2 d\left(y, S_{x_{i}}^{c}\right), d\left(y, y_{\mathrm{var}}\right)\right),
$$

so that we have:

$$
\begin{aligned}
|f(y)-f(\tilde{y})| & =\left|f_{x_{i}}(y)\right| \geq \frac{\alpha_{\mathrm{var}}}{2} d(y, \tilde{y}) \\
d(y, \tilde{y}) & \leq \varepsilon_{\mathrm{loss}} \leq \varepsilon .
\end{aligned}
$$




\section{BlOW-UPS OF DIFFERENTIABILITY SPACES}

\subsection{Blow-ups of differentiability spaces are differentiability spaces.}

Theorem 5.1. Let $(X, \mu)$ be a complete doubling metric measure space and assume that for $\mu$-a.e. $p$ there is some $(Y, \nu, q) \in \mathrm{Bw}-\mathrm{up}(X, \mu, p)$ which is not a differentiability space. Then $(X, \mu)$ is not a differentiability space.

Proof. In the following we will assume that $\mu(X)=1$. By Theorems 4.1, 4.8 it suffices to show that at $\mu$-a.e. point $p$ there is a sequence of "bad tiles" satisfying the assumption of Theorem 4.8.

Step 1: Choice of Christ's cubes.

In $(X, \mu)$ we choose a set of Christ's dyadic cubes [Chr90, HK12] $\mathcal{Q}$ using the scales $\left\{2^{-n}\right\}_{n}$. The properties of $Q$ that we will use are:

(Cube1): Cubes are open and there are constants $C_{\text {ann }} \geq 1$ and $\beta_{\text {ann }}>0$ such that for $\tau \in(0,1)$ and $Q \in \mathcal{Q}$ the "annulus"

$$
Q(\tau)=\left\{p \in Q: d\left(p, Q^{c}\right) \leq \tau \operatorname{diam} Q\right\}
$$

satisfies:

$$
\mu(Q(\tau)) \leq C_{\mathrm{ann}} \tau^{\beta_{\mathrm{ann}}} \mu(Q) .
$$

(Cube2): There is a $k_{\text {ann }} \geq 1$ such that each ball $B(p, r / 2)$, where $r \in$ $\left[2^{-n}, 2^{-n+1}\right]$, contains a cube $Q$ of generation $k_{\text {ann }}+n$ and the measures $\mu(Q)$ and $\mu(B(p, r))$ are uniformly comparable.

In the following, we will only consider blow-ups at points $p \in X$ where the conclusion of Theorem 3.18 holds.

Step 2: Uniformizing a bad function.

We now consider some $(Y, \nu, q) \in \operatorname{Bw}-u p(X, \mu, p)$ which is not a differentiability space. Note that $\nu$ is doubling with doubling constant bounded by $C_{\mu}^{4}, C_{\mu}$ being the doubling constant of $\mu$. Thus, the index of the module $X(\nu)$ is bounded by $\left\lceil\log _{2} C_{\mu}^{4}\right\rceil$. We fix the parameter $\varepsilon_{\tan , 1} \in(0,1)$ and use the argument of Lemma 4.17 in [Sch13] to show that there are a Borel subset $S_{\text {ndiff }} \subset Y$ with $\nu\left(S_{\text {ndiff }}\right)>0$ and a Lipschitz function $f: Y \rightarrow \mathbb{R}$ such that:

$$
\begin{gathered}
\operatorname{Lip} f(x) \in[1,2] \quad\left(\forall x \in S_{\text {ndiff }}\right) \\
|d f(x)|_{\mathcal{E}(\nu)} \leq \varepsilon_{\tan , 1} \quad\left(\forall x \in S_{\text {ndiff }}\right) .
\end{gathered}
$$

We choose a point $\tilde{q}$ that is a Lebesgue density point $\tilde{q}$ of $S_{\text {ndiff }}$, and an approximate continuity point of Lip $f$ and $|d f|_{\mathcal{E}(\nu)}$. By shifting the basepoint $\tilde{q}$ and rescaling $(Y, \nu, q)$ and $f$, we can assume that:

(BadF1): $S_{\text {ndiff }} \subset B_{Y}(q, 2)$ and $\nu\left(B_{Y}(q, 2) \backslash S_{\text {ndiff }}\right) \leq \varepsilon_{\text {tan }, 1}$;

(BadF2): The Lipschitz constant of the restriction of $f$ to $S_{\text {ndiff }}$ is at most 3 ;

(BadF3): For $\nu$-a.e. $x \in S_{\text {ndiff }}$ there is a sequence $\operatorname{Seq}(x)=\left\{x_{n}\right\} \subset S_{\text {ndiff }} \backslash\{x\}$ converging to $x$ such that $f$ witnesses at $x$ a definite amount of variation at scale $d\left(x, x_{n}\right)$ :

$$
\left|f(x)-f\left(x_{n}\right)\right| \geq\left(1-\varepsilon_{\tan , 1}\right) d\left(x, x_{n}\right)>0 .
$$

We now use MacShane's Lemma to extend $f \mid S_{\text {ndiff }}$ to a 3-Lipschitz function $\tilde{f}$ : $Y \rightarrow \mathbb{R}$. Note that (BadF3) and (5.5) remain valid replacing $f$ with $\tilde{f}$. In the following we will write $f$ for $\tilde{f}$. 
Step 3: Truncating the function $f$.

Fix parameters $\tau_{\text {ann }} \in(0,1)$ and $\tau_{\text {cut }} \in\left(0, \tau_{\text {ann }}\right)$. We fix $N \in \mathbb{N}$ and let

$$
\alpha \in[0,1) \cap \frac{1}{N^{2}} \mathbb{Z}
$$

we let:

$$
\psi_{\alpha}(\cdot)=d\left(\cdot, \frac{1}{N} \mathbb{Z}+\alpha\right),
$$

which is a 1-Lipschitz function. Using the pigeonhole principle (see [Bat12, Lemma 4.1] for details) we can find a value of $\alpha$ and a Borel set $S_{\text {var }} \subset S_{\text {ndiff }}$ such that:

$$
\nu\left(S_{\text {ndiff }} \backslash S_{\text {var }}\right) \leq \frac{4}{N} \nu\left(S_{\text {ndiff }}\right) \leq \frac{4}{N} \nu\left(B_{Y}(q, 2)\right),
$$

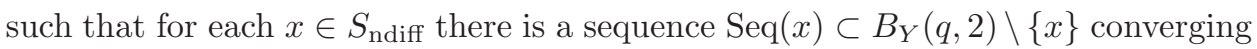
to $x$ such that, similarly as in (BadF3), one has:

$$
\left|\psi_{\alpha} \circ f(x)-\psi_{\alpha} \circ f\left(x_{n}\right)\right| \geq\left(1-\varepsilon_{\tan , 1}\right) d\left(x, x_{n}\right)>0 .
$$

Now note that the normalization

$$
\int_{B_{Y}(q, 1)}(1-d(p, x)) d \nu(x)=1
$$

implies

$$
\nu\left(B_{Y}\left(q, \frac{1}{2}\right)\right) \leq 2,
$$

and thus (5.9) leads to:

$$
\nu\left(S_{\text {ndiff }} \backslash S_{\text {var }}\right) \leq \frac{8 C_{\mu}^{8}}{N}
$$

Finally, note that:

$$
\begin{aligned}
\left\|\psi_{\alpha} \circ f\right\|_{\infty} & \leq \frac{1}{N} \\
\left|d\left(\psi_{\alpha} \circ f\right)\right|_{\mathcal{E}(\nu)} & \leq|d f|_{\mathcal{E}(\nu)} .
\end{aligned}
$$

We now fix a parameter $\varepsilon_{\tan , 2}>\varepsilon_{\tan , 1}$ and choose $N$ so that:

$\left(\right.$ BadC1): $\nu\left(B(q, 2) \backslash S_{\text {var }}\right) \leq \varepsilon_{\tan , 2}$ and $\left\|\psi_{\alpha} \circ f\right\|_{\infty} \leq \tau_{\text {cut }} ;$

(BadC2): For each $x \in S_{\text {var }}$ there is a sequence $\operatorname{Seq}(x)=\left\{x_{n}\right\}$ converging to $x$ such that

$$
\left|\psi_{\alpha} \circ f(x)-\psi_{\alpha} \circ f\left(x_{n}\right)\right| \geq\left(1-\varepsilon_{\tan , 2}\right) d\left(x, x_{n}\right)>0
$$

(BadC3): For each $x \in S_{\text {var }}$ we have $\left|d\left(\psi_{\alpha} \circ f\right)(x)\right|_{\mathcal{E}(\nu)} \leq \varepsilon_{\tan , 2}$.

In the following we will write $f$ for $\psi_{\alpha} \circ f$.

Step 4: Arranging convergence in $\ell^{\infty}$.

We now choose a sequence of rescalings $\lambda_{n} \nearrow \infty$ realizing $(Y, \nu, q)$, i.e.:

$$
\left(\lambda_{n} X=X_{n}, \mu_{n}, p\right) \stackrel{\mathrm{mGH}}{\longrightarrow}(Y, \nu, q) ;
$$

we can choose the convergence to take place in the container $\left(\ell^{\infty}, 0\right)$, and we will require that all basepoints map to 0 , but we will still distinguish them in the notation, i.e. we will denote the basepoint of $X_{n}$ by $p_{n}$. We now fix the parameter $\varepsilon_{\text {tan }, 3}>\varepsilon_{\text {tan }, 2}$. Then we can find $R_{\text {var }} \in\left(0, \tau_{\text {cut }}\right)$ and a compact set $K_{\text {Hau }} \subset B_{Y}(q, 2)$ such that: 
(Hau1): $\nu\left(B_{Y}(q, 2) \backslash K_{\text {Hau }}\right) \leq \varepsilon_{\tan , 3}$ and for each $x \in K_{\text {Hau }}$ one has

$$
|d f(x)|_{\mathcal{E}(\nu)} \leq \varepsilon_{\tan , 3}
$$

(Hau2): For each $x \in K_{\text {Hau }}$ there is an $x_{\mathrm{var}}=x_{\mathrm{var}}(x)$ satisfying:

$$
\begin{aligned}
\left|f(x)-f\left(x_{\mathrm{var}}\right)\right| & \geq\left(1-\varepsilon_{\mathrm{tan}, 3}\right) d\left(x, x_{\mathrm{var}}\right) \\
d\left(x, x_{\mathrm{var}}\right) & \in\left(R_{\mathrm{var}}, \tau_{\mathrm{cut}}\right) .
\end{aligned}
$$

Note that by MacShane's Lemma we can assume $f$ to be extended to a 3-Lipschitz $\operatorname{map} f: l^{\infty} \rightarrow \mathbb{R}$.

Step 5: Using the approximation scheme.

We fix the parameter $\varepsilon_{\text {cut }} \in\left(0, \varepsilon_{\tan , 3} R_{\mathrm{var}} / 2\right)$. Because of (Hau1) we can apply the Approximation Scheme Theorem 2.28 and find a function $\tilde{f}: \ell^{\infty} \rightarrow \mathbb{R}$ which is 3-Lipschitz, a compact set $K_{\text {app }} \subset K_{\text {Hau }}$, and an open set $U \supset K_{\text {app }}$ such that the following holds:

(App1): $\|\tilde{f}-f\|_{\infty} \leq \varepsilon_{\text {cut }}$ and $\nu\left(K_{\text {Hau }} \backslash K_{\text {app }}\right) \leq \varepsilon_{\text {cut }}$;

(App2): $U$ is an open set of $\ell^{\infty}$ contained in the closed $\varepsilon_{\text {cut }}$-neighbourhood $\bar{B}_{l \infty}\left(K_{\text {app }}, \varepsilon_{\text {cut }}\right)$ of $K_{\text {app }}$;

(App3): For each ball $B \subset U$ the restriction $\tilde{f} \mid B$ has Lipschitz constant $\leq \varepsilon_{\mathrm{cut}}+\varepsilon_{\mathrm{tan}, 3}$.

As $U$ is open and $\mu_{n} \stackrel{\mathrm{w}^{*}}{\longrightarrow} \nu$ we have by the properties of the weak* topology and the choice of basepoints that for $n$ sufficiently large:

$$
\int_{U \cap B_{\ell} \infty(0,1)}\left(1-d_{X_{n}}\left(p_{n}, x\right)\right) d \mu_{n}(x) \geq \int_{U \cap B_{\ell} \infty(0,1)}\left(1-d_{Y}(q, x)\right) d \nu(x)-\varepsilon_{\text {cut }} .
$$

We thus obtain:

$$
\begin{aligned}
\int_{U \cap B_{\ell} \infty(0,1)}\left(1-d_{X_{n}}\left(p_{n}, x\right)\right) d \mu_{n}(x) & \geq \int_{B_{\ell}(0,1)}\left(1-d_{Y}(q, x)\right) d \nu(x) \\
& -\nu\left(B_{Y}(q, 1) \backslash K_{\text {app }}\right)-\varepsilon_{\text {cut }} \\
& \geq 1-\left(\varepsilon_{\text {tan }, 3}+2 \varepsilon_{\text {cut }}\right) ;
\end{aligned}
$$

moreover, as on $B_{\ell \infty}(0,1 / 2) \cap X_{n}=B_{X_{n}}\left(p_{n}, 1 / 2\right)$ we have that $1-d_{X_{n}}\left(p_{n}, \cdot\right)$ is at least $1 / 2$, we conclude that:

$$
\mu_{n}\left(B_{X_{n}}\left(p_{n}, \frac{1}{2}\right) \backslash U\right) \leq 2\left(\varepsilon_{\tan , 3}+2 \varepsilon_{\text {cut }}\right) .
$$

Now for $x \in U \cap B_{X_{n}}\left(p_{n}, 1\right)$ we have:

$$
|d \tilde{f}(x)|_{\varepsilon\left(\mu_{n}\right)} \leq \varepsilon_{\mathrm{cut}}+\varepsilon_{\mathrm{tan}, 3}
$$

Moreover, we have:

$$
\|\tilde{f}\|_{\infty} \leq \varepsilon_{\text {cut }}+\tau_{\text {cut }},
$$

and for $x \in K_{\text {app }}$ we can find $x_{\text {var }}$ satisfying (5.18).

Step 6: Lifting the variation.

We now choose a parameter $\varepsilon_{\text {dens }}>0$ and a finite $\varepsilon_{\text {dens }}$-net $\mathcal{N}$ in $K_{\text {app }} \cap \bar{B}_{Y}(q, 1)$. For each $x \in \mathcal{N}$ we can find $x_{\mathrm{var}}(x) \in B_{Y}(q, 2)$ satisfying (5.18). We can thus construct the finite set:

$$
\mathcal{N}_{\mathrm{var}}=\left\{x_{\mathrm{var}}(x)\right\}_{x \in \mathcal{N}}
$$


and find a map $V: \mathcal{N} \rightarrow \mathcal{N}_{\text {var }}$ which associates $x_{\text {var }}(x)$ to $x$. Using (3.3), for $n$ sufficiently large, we can find a finite set

$$
\mathcal{N}_{n} \subset U \cap B_{X_{n}}\left(p_{n}, 1+\varepsilon_{\text {dens }}\right)
$$

of the same cardinality as $\mathcal{N}$ and a bijection $J_{n}: \mathcal{N} \rightarrow \mathcal{N}_{n}$ such that:

$$
d\left(J_{n}(x), x\right)<\varepsilon_{\text {dens }} .
$$

Similarly, for $n$ sufficiently large, we can also find a finite set

$$
\mathcal{N}_{n, \operatorname{var}} \subset U \cap B_{X_{n}}\left(p_{n}, 2+\varepsilon_{\text {dens }}\right)
$$

of the same cardinality as $\mathcal{N}_{\text {var }}$ and a bijection $J_{n, \text { var }}: \mathcal{N}_{\text {var }} \rightarrow \mathcal{N}_{n \text {,var }}$ such that:

$$
d\left(J_{n, \operatorname{var}}(x), x\right)<\varepsilon_{\mathrm{dens}} .
$$

We finally let $V_{n}=J_{n, \operatorname{var}} \circ V \circ J_{n}^{-1}$. For $y \in U \cap B_{X_{n}}\left(p_{n}, 1\right)$ we can find $x \in \mathcal{N}$ such that:

$$
d(y, x)=O\left(\varepsilon_{\mathrm{cut}}, \varepsilon_{\mathrm{dens}}\right) ;
$$

using that $\tilde{f}$ is 3-Lipschitz we obtain:

$$
\left|\tilde{f}(y)-\tilde{f}\left(V_{n}(x)\right)\right|=\left|\tilde{f}\left(J_{n}^{-1}(x)\right)-\tilde{f}\left(V \circ J_{n}^{-1}(x)\right)\right|+O\left(\varepsilon_{\text {cut }}, \varepsilon_{\text {dens }}\right) ;
$$

using the properties of $V$ we also conclude that:

$$
d\left(y, V_{n}(x)\right)=d\left(J_{n}^{-1}(x), V \circ J_{n}^{-1}(x)\right)+O\left(\varepsilon_{\text {cut }}, \varepsilon_{\text {dens }}\right) .
$$

Note that the constants hidden in the $O(\cdot)$ notation in (5.29)-(5.31) do not depend on $n$. As the parameters $\varepsilon_{\text {cut }}$ and $\varepsilon_{\text {dens }}$ are chosen after $R_{\mathrm{var}}$ and $\varepsilon_{\mathrm{tan}, 3}$, one can choose them sufficiently small so that:

$$
\begin{aligned}
\left|\tilde{f}(y)-\tilde{f}\left(V_{n}(x)\right)\right| & \geq\left(1-\varepsilon_{\tan , 3}\right) d\left(y, V_{n}(x)\right) \\
d\left(y, V_{n}(x)\right) & \in\left(R_{\text {var }} / 2,2 \tau_{\text {cut }}\right) .
\end{aligned}
$$

Step 7: Constructing the tiles.

Let $Q \in \mathcal{Q}$ be a dyadic cube of generation $g_{n}=\left\lfloor\log _{2} \lambda_{n}\right\rfloor+k_{\text {ann }}$ contained in $B_{X_{n}}\left(p_{n}, 1 / 2\right)$. For the moment we will compute distances using the rescaled metric. The cube $Q$ will be used to construct the tile. Specifically, recall that $Q$ is open and consider a parameter $\tau_{\text {ann }} \in(0,1 / 8)$ to be chosen later. The set $\tilde{Q}$ that we will use for the tile will be the closure in $X_{n}$ of $Q \backslash Q\left(\tau_{\text {ann }}\right)$. Now (Cube1) and (Cube2) imply that there is a uniform constant $C$ such that:

$$
\begin{aligned}
\operatorname{diam} \tilde{Q} & \approx_{C} \operatorname{diam} Q \approx_{C} 2^{-k_{\mathrm{ann}}} \\
\mu_{n}(\tilde{Q}) \approx_{C} \mu_{n}(Q) & \gtrsim_{C} \mu_{n}\left(B_{X_{n}}\left(p_{n}, 1\right)\right) ;
\end{aligned}
$$

this will give $(\mathbf{T} 1)$ and $(\mathbf{T} 2)$. Consider $Q\left(\tau_{\text {ann }}\right)$ and a $\frac{C 2^{k_{\text {ann }}}}{\tau_{\text {ann }}}$-Lipschitz function $\psi_{Q}$ which takes values in $[0,1]$ and such that:

$$
\begin{aligned}
\psi_{Q} & = \begin{cases}1 & \text { on } Q \backslash Q\left(2 \tau_{\text {ann }}\right) \\
0 & \text { on } \tilde{Q}^{c}\end{cases} \\
\left|\psi_{Q}(x)\right| \leq & \frac{C 2^{k_{\text {ann }}}}{\tau_{\text {ann }}} d\left(x, \tilde{Q}^{c}\right) .
\end{aligned}
$$


Let $h=\psi_{Q} \tilde{f}$; using equation (5.23) we conclude that $h$ has Lipschitz constant at most

$$
3+\frac{C 2^{k_{\text {ann }}}}{\tau_{\text {ann }}}\left(\tau_{\text {cut }}+\varepsilon_{\text {cut }}\right)
$$

As the parameters $\tau_{\text {cut }}$ and $\varepsilon_{\text {cut }}$ are chosen after $k_{\text {ann }}$ and $\tau_{\text {ann }}$ have been determined, we can choose them small enough to ensure that $h$ is 4 -Lipschitz. We then also have:

$$
|h(x)| \leq \frac{C 2^{k_{\text {ann }}}}{\tau_{\text {ann }}}\left(\tau_{\text {cut }}+\varepsilon_{\text {cut }}\right) d\left(x, \tilde{Q}^{c}\right) \leq 4 d\left(x, \tilde{Q}^{c}\right) .
$$

The function $h$ will be the function that we use to construct the tile. Now (T3) follows from (5.35), (5.36). We now let:

$$
Q_{\text {ndiff }}=Q \backslash Q\left(2 \tau_{\text {ann }}+2 \tau_{\text {cut }}\right) \cap U \subset \tilde{Q} \subset B_{X_{n}}\left(p_{n}, 1 / 2\right) ;
$$

we then have, using (Cube1) and minding (5.21):

$$
\begin{aligned}
\mu_{n}\left(\tilde{Q} \backslash Q_{\text {ndiff }}\right) & \leq \mu_{n}\left(Q\left(2 \tau_{\text {ann }}+2 \tau_{\text {cut }}\right)\right)+\mu_{n}\left(B_{X_{n}}\left(p_{n}, 1 / 2\right) \backslash U\right) \\
& \leq C_{\text {ann }}\left(2 \tau_{\text {ann }}+2 \tau_{\text {cut }}\right)^{\beta_{\text {ann }}} \mu_{n}(Q)+\mu_{n}\left(B_{X_{n}}\left(p_{n}, 1 / 2\right) \backslash U\right) \\
& \leq C_{\text {ann }} C\left(2 \tau_{\text {ann }}+2 \tau_{\text {cut }}\right)^{\beta_{\text {ann }}} \mu_{n}(\tilde{Q})+2\left(\varepsilon_{\text {tan }, 3}+2 \varepsilon_{\text {cut }}\right) .
\end{aligned}
$$

As the parameters on the right hand side of (5.38) are chosen after Step 1, given a fixed $\varepsilon_{\text {loss }}>0$ we chan choose those parameters so that:

$$
\mu_{n}\left(\tilde{Q} \backslash Q_{\text {ndiff }}\right) \leq \varepsilon_{\text {loss }} \mu_{n}(\tilde{Q}) .
$$

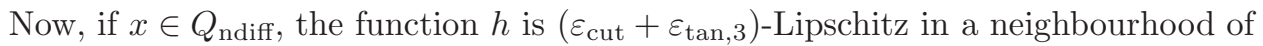
$x$ and hence:

$$
|d h(x)|_{\varepsilon_{\left(\mu_{n}\right)} \leq\left(\varepsilon_{\mathrm{cut}}+\varepsilon_{\mathrm{tan}, 3}\right) .}
$$

Given $\varepsilon_{\text {grad }}$ we can choose $\varepsilon_{\text {cut }}$ and $\varepsilon_{\tan , 3}$ so that their sum is $\leq \varepsilon_{\text {grad }}$; this gives (T4). Also, given $x \in Q_{\text {ndiff }}$ we can find by (5.32) a point $y \in Q \backslash Q\left(\tau_{\text {ann }}\right) \subset \tilde{Q}$ such that:

$$
\begin{aligned}
|h(x)-h(y)| & \geq\left(1-2 \varepsilon_{\tan , 3}\right) d(x, y)>0 \\
d(x, y) & \leq 2 \tau_{\text {cut }} .
\end{aligned}
$$

Choosing $\tau_{\text {cut }}$ sufficiently small we can ensure that

$$
d(x, y) \leq \varepsilon_{\text {loss }} .
$$

Finally, choosing $\varepsilon_{\tan , 3}$ sufficiently small we can also ensure that:

$$
1-2 \varepsilon_{\tan , 3} \geq \frac{2}{3}
$$

thus (5.41)-(5.43) give (T5). Rescaling $h$ back to $X$ (i.e. $h \mapsto \lambda_{n}^{-1} h$ ), we conclude that $(\tilde{Q}, h)$ is an:

$$
\left[\max (C, 5), \frac{2}{3}, \varepsilon_{\text {grad }}, \varepsilon_{\text {loss }}, \lambda_{n}^{-1}\right] \text {-tile }
$$

at $p$. 


\subsection{Independence of the $p$-weak gradient on $p$.}

Definition 5.45. Let $I \subset \mathbb{R}$ be a nondegenerate closed interval and $A_{I}: \mathbb{R} \rightarrow \mathbb{R}$ the unique orientation-preserving affine map which maps $[0,1]$ onto $I$. For $\varepsilon>0$ let:

$$
\operatorname{Slide}(I, \varepsilon)=\{\gamma \in \operatorname{Curves}(X): \forall t \in[0, \varepsilon] \quad t+I \subset \operatorname{dom} \gamma\}
$$

let:

$$
\operatorname{Slide}_{I, \varepsilon}: \operatorname{Slide}(I, \varepsilon) \times[0, \varepsilon] \rightarrow \operatorname{Curves}(X,[0,1])
$$

be the map such that:

$$
\operatorname{Slide}_{I, \varepsilon}(\gamma, t)(s)=\gamma\left(A_{I}(s)+t\right) .
$$

Lemma 5.49 (Test plan associated with an Alberti representation). Let $\mathcal{A}=[Q, 1]$ be an Alberti representation of a measure $\nu$ such that for some $C_{\nu}>0$ one has $\nu \leq C_{\nu} \mu$. Assume that for some closed interval $I, \varepsilon>0$ and $C_{0}>0$ the measure $Q$ is concentrated on the set of $C_{0}$-Lipschitz curves in $\operatorname{Slide}(I, \varepsilon)$. Assuming that $Q$ is a probability measure, we can associate to $\mathcal{A}$ a probability $\pi$ on $\operatorname{Curves}(X ;[0,1])$ by:

$$
\pi=\operatorname{Slide}_{I, \varepsilon, \#}\left(Q \times \frac{1}{\varepsilon} \mathcal{L}^{1}\llcorner[0, \varepsilon]) .\right.
$$

Then for any $q \in[1, \infty)$, $\pi$ defines a $q$-test plan.

Proof. Note that for $Q$-a.e. $\gamma$ one has that $\operatorname{md} \gamma \leq C_{0}$ holds $\mathcal{L}^{1}\llcorner\operatorname{dom} \gamma$-a.e. Now the derivative of $A_{I}$ is $\mathcal{L}^{1}(I)$ and so for $\pi$-a.e. $\gamma$ one has that md $\gamma \leq C_{0} \mathcal{L}^{1}(I)$ holds $\mathcal{L}^{1}\llcorner\operatorname{dom} \gamma$-a.e.; one thus gets:

$$
\int d \pi(\gamma) \int_{0}^{1}(\operatorname{md} \gamma(t))^{q} d t \leq\left(C_{0} \mathcal{L}^{1}(I)\right)^{q},
$$

which gives (2.43). Let $\varphi$ be a nonnegative continuous function of $X$; then:

$$
\begin{aligned}
\int \varphi d \operatorname{Ev}_{t \#} \pi & =\int \varphi(\gamma(t)) d \pi(\gamma)=\int d Q(\gamma) \frac{1}{\varepsilon} \int_{0}^{\varepsilon} \varphi\left(\gamma\left(A_{I}(t)+s\right)\right) d s \\
& \leq \frac{1}{\varepsilon} \int \varphi d \nu \leq \frac{C_{\nu}}{\varepsilon} \int \varphi d \mu,
\end{aligned}
$$

which establishes (2.44).

Definition 5.53 (Regular Alberti representation). An Alberti representation $[Q, 1]$ is regular if $Q$ is concentrated on the set of unit-speed geodesic lines of $X$. Here we think of unit-speed geodesic lines as maps $\gamma: \mathbb{R} \rightarrow X$, and so they have infinite length.

Lemma 5.54. Let $[Q, 1]$ be a regular Alberti-representation of $\mu$ and $(x, r) \in X \times$ $(0, \infty)$; then:

$$
Q\left(\left\{\gamma: \gamma^{-1}(B(x, r)) \neq \emptyset\right\}\right) \leq \frac{\mu(B(x, 2 r))}{r} .
$$

Proof. It suffices to observe that if $\gamma$ is a unit-speed geodesic line in $X$ then $\gamma^{-1}(B(x, r)) \neq \emptyset$ implies:

$$
\mathcal{L}^{1}\left(\gamma^{-1}(B(x, 2 r))\right) \geq r
$$


Definition 5.57. A differentiability space $(X, \mu)$ is regular if:

(Reg1): The measure $\mu$ is doubling and there is a unique differentiability chart $\left(X, \Phi=\left\{\varphi_{i}\right\}_{i=1}^{n}\right)$ such that $\Phi: X \rightarrow \mathbb{R}^{n}$ is 1-Lipschitz;

(Reg2): The local norm $|\cdot|_{\mathcal{E}(\mu)}$ is constant;

(Reg3): For each vector $v \in \mathcal{X}(\mu)$ (note that we can canonically identify a vector in the measurable tangent bundle with a derivation, as the chart is global) with $|v|_{\mathcal{X}(\mu)}=1$ there is an Alberti representation $\left[Q_{v}, 1\right]$ of $\mu$ where $Q_{v}$ is concentrated on the set of unit-speed lines $\gamma$ in $X$ satisfying:

$$
(\Phi \circ \gamma)^{\prime}=v
$$

Lemma 5.59 (Iterated blow-ups are regular). Let $(X, \mu)$ be a differentiability space. Then for $\mu$-a.e. $x$ there is an integer $N(x)$ such that, whenever $(Y, \nu, y) \in$ $\mathrm{Bw}-\mathrm{up}(X, \mu, x)$, up to passing to at most $N(x)$ iterated blow-ups, i.e. up to replacing $(Y, \nu, y)$ with $\left(Y_{l}, \nu_{l}, y_{l}\right)$ where:

$$
\begin{aligned}
\left(Y_{i}, \nu_{i}, y_{i}\right) & \in \mathrm{Bw}-u p\left(Y_{i-1}, \nu_{i-1}, y_{i-1}\right) \quad 1 \leq i \leq l \leq N(x) \\
\left(Y_{0}, \nu_{0}, y_{0}\right) & =(Y, \nu, y),
\end{aligned}
$$

one can assume that $(Y, \nu, y)$ is a regular differentiability space. The integer $N(x)$ satisfies:

$$
N(x) \leq\left\lfloor\log _{2} C_{\mu}(x)\right\rfloor,
$$

where $C_{\mu}(x)$ is the asymptotically doubling constant of $\mu$ at $X$. In particular, if $X$ has finite Assoaud dimension $N$, then

$$
N(x) \leq N .
$$

Proof. At $\mu$-a.e. $x$ the conclusion of Theorem 3.18 holds, and each $(Y, \nu, y) \in$ $\operatorname{Bw}-\operatorname{up}(X, \mu, x)$ is a differentiability space. Now by (IndBound) in Theorem 2.26 the index of $X(\nu)$ is uniformly bounded by some $N(x)$ which satisfies (5.61), (5.62). Thus in passing to iterated blow-ups as in (5.60), the differentiability dimension can increase at most $N(x)$ times. If $l$ is the smallest integer such that in passing from $\left(Y_{l-1}, \nu_{l-1}, y_{l-1}\right)$ to $\left(Y_{l}, \nu_{l}, y_{l}\right)$ the differentiability dimension does not increase, then $\left(Y_{l}, \nu_{l}, y_{l}\right)$ is regular by Theorem 3.30 (compare also [CKS15]).

Theorem 5.63 (The $p$-weak gradient does not depend on $p$ ). Let $(X, \mu)$ be a regular differentiability space and $f$ a Lipschitz function on $X$; then for any $p \in(1, \infty)$ :

$$
|\nabla f|_{p, \mathrm{w}}=\operatorname{Lip} f \quad \mu \text {-a.e. }
$$

Proof. Step 1: Uniformization.

Let $g$ be a $p$-weak upper gradient of $f$ and let $A_{\text {cont }}$ denote the set of differentiability points of $f$ and of approximate continuity points of $d f, g$ and $\operatorname{Lip} f$. We fix $x \in A_{\text {cont }}$ and choose the parameter $\varepsilon_{\text {cont }}>0$. Up to rescaling $f$ and $g$ we can assume that:

$$
|d f(x)|_{\mathcal{E}(\mu)}=\operatorname{Lip} f(x)=1 .
$$

We let $v \in X(\mu)$ be a unit vector where $d f(x)$ attains the norm and let:

$$
A_{x}=\left\{y \in A_{\text {cont }}:|d f(y)-d f(x)| \leq \varepsilon_{\text {cont }} \text { and }|g(y)-g(x)| \leq \varepsilon_{\text {cont }}\right\} .
$$

Note that for $r$ sufficiently small we can assume:

$$
\mu\left(B(x, r) \backslash A_{x}\right) \leq \varepsilon_{\mathrm{cont}} \mu(B(x, r)) .
$$


Step 2: Construction of a q-test plan.

Let $q$ be the dual exponent of $p$. Let $[Q, 1]$ be an Alberti representation of $\mu$ as in the definition of a regular differentiability space where we use the unit vector $v$. Let

$$
\Gamma_{x, r}=\left\{\gamma \text { is a unit speed line with } \gamma^{-1}(B(x, r)) \neq \emptyset\right\}
$$

letting $Q_{x, r}=Q\left\llcorner\Gamma_{x, r}\right.$ we have by Lemma 5.54:

$$
\left\|Q_{x, r}\right\| \leq \frac{\mu(B(x, 2 r))}{r} .
$$

Let $\mu_{x, r}$ be the measure corresponding to the Alberti representation $\left[Q_{x, r}, 1\right]$ and note that:

$$
\begin{aligned}
\mu_{x, r} & \leq \mu \\
\frac{d \mu_{x, r}}{d \mu} & =1 \quad \text { on } B(x, r) .
\end{aligned}
$$

We now let:

$$
\Gamma_{\text {short }}=\left\{\gamma \text { is a unit speed line with } \gamma^{-1}\left(A_{x} \cap B(x, r)\right) \leq \varepsilon_{\text {short }} r\right\} \text {; }
$$

we then obtain the estimates:

$$
\begin{aligned}
\mu_{x, r}\left(A_{x} \cap B(x, r)\right) \geq\left(1-\varepsilon_{\text {cont }}\right) \mu(B(x, r)) \\
\mu_{x, r}\left(A_{x} \cap B(x, r)\right) \leq \int_{\Gamma_{\text {short }}} \mathcal{L}^{1}\left(\gamma^{-1}\left(A_{x} \cap B(x, r)\right)\right) d Q_{x, r}(\gamma) \\
+\int_{\Gamma_{\text {short }}^{c}} \mathcal{L}^{1}\left(\gamma^{-1}\left(A_{x} \cap B(x, r)\right)\right) d Q_{x, r}(\gamma) \\
\leq \varepsilon_{\text {short }} r Q_{x, r} \| \\
+\int_{\Gamma_{\text {short }}^{c}} \mathcal{L}^{1}\left(\gamma^{-1}\left(A_{x} \cap B(x, r)\right)\right) d Q_{x, r}(\gamma) \\
\leq \varepsilon_{\text {short }} \mu(B(x, 2 r)) \\
+\int_{\Gamma_{\text {short }}^{c}} \mathcal{L}^{1}\left(\gamma^{-1}\left(A_{x} \cap B(x, r)\right)\right) d Q_{x, r}(\gamma) ;
\end{aligned}
$$

combining (5.72), (5.73) we conclude that for $\varepsilon_{\text {short }}$ sufficiently small:

$$
\int_{\Gamma_{\text {short }}^{c}} \mathcal{L}^{1}\left(\gamma^{-1}\left(A_{x} \cap B(x, r)\right)\right) d Q_{x, r}(\gamma)>0
$$

Using a measurable selection principle (see [Kec95, Chap. 18]) we can find an Alberti representation $\left[Q_{A_{x}}, 1\right]$ of a measure $\nu \ll \mu$ such that:

(Test1): $Q_{A_{x}}$ is a finite Radon measure, $\frac{d \nu}{d \mu} \leq 1$;

(Test2): For $Q_{A_{x}}$-a.e. $\gamma$, letting $I=[0,3 r]$ we have for each $t \in[0, r]$ :

$$
\begin{aligned}
I+t & \subset \operatorname{dom} \gamma \\
\mathcal{L}^{1}\left(\gamma^{-1}\left(A_{x} \cap B(x, r)\right) \cap(I+t)\right) & >0 ;
\end{aligned}
$$

(Test3): $Q_{A_{x}}$-a.e. $\gamma$ is a unit-speed geodesic segment of length at most $6 r$. 
Using Lemma 5.49 (and setting $\varepsilon=r$ ) we can associate to $\left[Q_{A_{x}} /\left\|Q_{A_{x}}\right\|, 1\right]$ a $q$-test plan $\pi$.

Step 3: Applying Lebesgue's differentiation along curves.

As $g \in L^{p}(\mu)$, conditions (Test1)-(Test3) imply that for $\pi$-a.e. $\gamma$ there is a nondegenerate interval $J_{\gamma} \subset \operatorname{dom} \gamma$ such that $g \circ \gamma \in L^{1}\left(\mathcal{L}^{1}\left\llcorner J_{\gamma}\right)\right.$ and $J_{\gamma}$ meets $\gamma^{-1}\left(A_{x}\right)$ in positive Lebesgue measure. Moreover, for $\pi$-a.e. $\gamma$ for each $(a, b) \subset J_{\gamma}$ we also have that:

$$
|f \circ \gamma(b)-f \circ \gamma(b)| \leq \int_{a}^{b} g \circ \gamma d \mathcal{L}^{1}
$$

if $t_{0} \in J_{\gamma}$ is an interior point of $J_{\gamma}$, a differentiability point of $f \circ \gamma$, and a Lebesgue point of $\gamma^{-1}\left(A_{x}\right)$, applying Lebesgue's differentiation at $t_{0}$ we obtain:

$$
g\left(\gamma\left(t_{0}\right)\right) \geq\left\langle d f\left(\gamma\left(t_{0}\right)\right), v\right\rangle
$$

which implies

$$
g(x) \geq 1-2 \varepsilon_{\text {cont }} .
$$

Remark 5.79. Theorem 5.63 has a counterpart in the category of functions of bounded variations; i.e. one can show that in a regular differentiability space the total variation measure of a Lipschitz function $f$ coincides with $\operatorname{Lip} f \cdot \mu$; we omit the details because they are mainly technical and do not add much mathematical substance to the paper.

\section{REFERENCES}

[ACM12] Luigi Ambrosio, Maria Colombo, and Simone Di Marino. Sobolev spaces in metric measure spaces: reflexivity and lower semicontinuity of slope. Advanced Studies in Pure Mathematics, 2012.

[ACP05] Giovanni Alberti, Marianna Csörnyei, and David Preiss. Structure of null sets in the plane and applications. In European Congress of Mathematics, pages 3-22. Eur. Math. Soc., Zürich, 2005.

[ACP10] Giovanni Alberti, Marianna Csörnyei, and David Preiss. Differentiability of Lipschitz functions, structure of null sets, and other problems. In Proceedings of the International Congress of Mathematicians. Volume III, pages 1379-1394, New Delhi, 2010. Hindustan Book Agency.

[ADM14] Luigi Ambrosio and Simone Di Marino. Equivalent definitions of $B V$ space and of total variation on metric measure spaces. J. Funct. Anal., 266(7):4150-4188, 2014.

[AGS13] Luigi Ambrosio, Nicola Gigli, and Giuseppe Savaré. Density of Lipschitz functions and equivalence of weak gradients in metric measure spaces. Rev. Mat. Iberoam., 29(3):969996, 2013.

[Alb93] Giovanni Alberti. Rank one property for derivatives of functions with bounded variation. Proc. Roy. Soc. Edinburgh Sect. A, 123(2):239-274, 1993.

[Bat12] D. Bate. Structure of measures in Lipschitz differentiability spaces (v2). ArXiv e-prints, August 2012.

[Bat15] David Bate. Structure of measures in Lipschitz differentiability spaces. J. Amer. Math. Soc., 28(2):421-482, 2015.

[BL15] D. Bate and S. Li. The geometry of Radon-Nikodym Lipschitz differentiability spaces. ArXiv e-prints, May 2015.

[BS13] David Bate and Gareth Speight. Differentiability, porosity and doubling in metric measure spaces. Proc. Amer. Math. Soc., 141(3):971-985, 2013.

[Che99] J. Cheeger. Differentiability of Lipschitz functions on metric measure spaces. Geom. Funct. Anal., 9(3):428-517, 1999.

[Chr90] Michael Christ. A $T(b)$ theorem with remarks on analytic capacity and the Cauchy integral. Colloq. Math., 60/61(2):601-628, 1990. 
[CK09] Jeff Cheeger and Bruce Kleiner. Differentiability of Lipschitz maps from metric measure spaces to Banach spaces with the Radon-Nikodým property. Geom. Funct. Anal., 19(4):1017-1028, 2009.

[CK10a] Jeff Cheeger and Bruce Kleiner. Differentiating maps into $L^{1}$, and the geometry of BV functions. Ann. of Math. (2), 171(2):1347-1385, 2010.

[CK10b] Jeff Cheeger and Bruce Kleiner. Metric differentiation, monotonicity and maps to $L^{1}$. Invent. Math., 182(2):335-370, 2010.

[CK13] Jeff Cheeger and Bruce Kleiner. Realization of metric spaces as inverse limits, and bilipschitz embedding in $L_{1}$. Geom. Funct. Anal., 23(1):96-133, 2013.

[CKN11] Jeff Cheeger, Bruce Kleiner, and Assaf Naor. Compression bounds for Lipschitz maps from the Heisenberg group to $L_{1}$. Acta Math., 207(2):291-373, 2011.

[CKS15] J. Cheeger, B. Kleiner, and A. Schioppa. Infinitesimal structure of differentiability spaces, and metric differentiation. ArXiv e-prints, March 2015.

[CM97] Tobias H. Colding and William P. Minicozzi, II. Harmonic functions on manifolds. Ann. of Math. (2), 146(3):725-747, 1997.

[DS15] S. Di Marino and G. Speight. The $p$-Weak Gradient Depends on p. Proc. Amer. Math. Soc., 143(11):5239-5252, 2015.

[GMR15] Nicola Gigli, Andrea Mondino, and Tapio Rajala. Euclidean spaces as weak tangents of infinitesimally Hilbertian metric measure spaces with Ricci curvature bounded below. $J$. Reine Angew. Math., 705:233-244, 2015.

[HK98] Juha Heinonen and Pekka Koskela. Quasiconformal maps in metric spaces with controlled geometry. Acta Math., 181(1):1-61, 1998.

[HK12] Tuomas Hytönen and Anna Kairema. Systems of dyadic cubes in a doubling metric space. Colloq. Math., 126(1):1-33, 2012.

[Kec95] Alexander S. Kechris. Classical descriptive set theory, volume 156 of Graduate Texts in Mathematics. Springer-Verlag, New York, 1995.

[Kei02] Stephen John Keith. A differentiable structure for metric measure spaces. ProQuest LLC, Ann Arbor, MI, 2002. Thesis (Ph.D.)-University of Michigan.

[Kei03] Stephen Keith. Modulus and the Poincaré inequality on metric measure spaces. Math. Z., 245(2):255-292, 2003.

[Kei04] Stephen Keith. A differentiable structure for metric measure spaces. Adv. Math., 183(2):271-315, 2004.

[KM98] Pekka Koskela and Paul MacManus. Quasiconformal mappings and Sobolev spaces. Studia Math., 131(1):1-17, 1998.

[KM11] B. Kleiner and J. Mackay. Differentiable structures on metric measure spaces: A Primer. ArXiv e-prints, August 2011. To appear in Ann. Sc. Norm. Super. Pisa Cl. Sci.

[LD11] Enrico Le Donne. Metric spaces with unique tangents. Ann. Acad. Sci. Fenn. Math., 36(2):683-694, 2011.

[Pre87] David Preiss. Geometry of measures in $\mathbf{R}^{n}$ : distribution, rectifiability, and densities. Ann. of Math. (2), 125(3):537-643, 1987.

[Sch13] Andrea Schioppa. Derivations and Alberti representations. ArXiv e-prints, November 2013.

[Sch14a] A. Schioppa. Metric Currents and Alberti representations. ArXiv e-prints, March 2014.

[Sch14b] Andrea Schioppa. Derivations and Alberti representations. ProQuest LLC, Ann Arbor, MI, 2014. Thesis (Ph.D.)-New York University.

[Sch14c] Andrea Schioppa. On the relationship between derivations and measurable differentiable structures. Ann. Acad. Sci. Fenn. Math., 39(1):275-304, 2014.

[Sch15] A. Schioppa. The Poincaré Inequality does not improve with blow-up. ArXiv e-prints, April 2015

[Sha00] Nageswari Shanmugalingam. Newtonian spaces: an extension of Sobolev spaces to metric measure spaces. Rev. Mat. Iberoamericana, 16(2):243-279, 2000.

[Wea00] Nik Weaver. Lipschitz algebras and derivations. II. Exterior differentiation. J. Funct. Anal., 178(1):64-112, 2000.

ETHZ

E-mail address: andrea.schioppa@math.ethz.ch 